\title{
Dynamical treatment of Fermi motion in a microscopic description of heavy ion collisions
}

\author{
G. Peilert, J. Konopka, H. Stöcker, and W. Greiner \\ Institut für Theoretische Physik, J. W. Goethe-Universität, D-6000 Frankfurt am Main, Germany \\ M. Blann and M. G. Mustafa \\ Lawrence Livermore National Laboratory, P.O. Box 808, Livermore, California 94550 \\ (Received 14 January 1992)
}

\begin{abstract}
A quasiclassical Pauli potential is used to simulate the Fermi motion of nucleons in a molecular dynamical simulation of heavy ion collisions. The thermostatic properties of a Fermi gas with and without interactions are presented. The inclusion of this Pauli potential into the quantum molecular dynamics (QMD) approach yields a model with well defined fermionic ground states, which is therefore also able to give the excitation energies of the emitted fragments. The deexcitation mechanisms (particle evaporation and multifragmentation) of the new model are investigated. The dynamics of the QMD with Pauli potential is tested by a wide range of comparisons of calculated and experimental doubledifferential cross sections for inclusive $p$-induced reactions at incident energies of 80 to $160 \mathrm{MeV}$. Results at 256 and $800 \mathrm{MeV}$ incident proton energy are presented as predictions for completed experiments which are as yet unpublished.

PACS number(s): 24.10.Cn, 25.70.- z
\end{abstract}

\section{INTRODUCTION}

The study of the production and decay of hot and compressed nuclear systems has gained great interest in recent years. This is due to the wealth of physical processes, which are involved in such reactions. This field was opened for detailed experimental investigations with the construction of heavy ion accelerators, where pieces of nuclear matter far from their ground states could be produced for the first time in the laboratory. One of the main goals of such experiments has been to study the nuclear equation of state at high density [1-17], and at low density, where a liquid-gas phase transition is expected [18-24]. In low-energy heavy ion collisions or highenergy proton-induced reactions this phase transition results in an increase of the total entropy [24] and in the fragmentation of the system [25]. While the entropy is not directly measurable in heavy ion experiments, the formation of intermediate mass fragments (IMFs) can be observed directly. This question was first addressed experimentally via inclusive proton-induced reactions [26] and later in heavy ion reactions [11,27-38].

All the inclusive, i.e., impact-parameter-averaged, experiments have shown a typical power-law dependence $\sigma(A) \approx A^{-\tau}$ of the mass yield distributions, where the critical exponent $\tau$ was compatible with a value of $\approx 2.5$. Fisher [25] made such a prediction for fragmentations resulting from a classical liquid-vapor phase transition only at the critical point. The experimental results therefore would have this strange result that the phase transition is only observed at the critical point, the more characteristic distribution for liquid-vapor phase transition; i.e., an exponential with fractional power in the argument is not observed in inclusive data. However, we have shown theoretically $[21,72]$ that for central collisions the fragment yield is indeed exponential. This theoretical result is experimentally investigated at the $4 \pi$ facility at the Gesellschaft für Schwerionenforschung (GSI).

The major shortcoming of those experiments was, however, that no selection on multiplicity (impact parameter) could be done, and so the results could also be of geometrical or other origin and not a signature of a phase transition. In recent years it has become possible to do exclusive measurements of multifragmentation reactions. This has been done with streamer chamber detectors $[33,34]$ or with electronic $4 \pi$ detectors [11,35-39]. With the availability of $4 \pi$ detectors it is possible to study the multifragment breakup of nuclear systems in much greater detail, due to the huge number of events which can be sampled.

A lot of progress has also been made in theoretical descriptions of the multifragmentation process of hot, compressed nuclei. On the one hand, there exist a variety of models that treat this process in a statistical way in microcanonical, canonical, or grandcanonical ensembles [40-45], or by means of evaporation models [46-49]. These models, however, start with a totally equilibrated system. While this may be a good approximation in heavy ion reactions at very low energies (around the Coulomb barrier the projectile may excite the collective degrees of freedom of the target) it is still an open question whether at higher energies a global, or even a local, equilibrium is achieved (see also Ref. [74]).

On the other hand, many models exist which describe heavy ion reactions microscopically in terms of the dynamics of the interacting nucleons. The most common models of this type are the VUU/BUU [50-59], molecular dynamics [60-81] (in which classical many-body correlations are retained), and CASCADE type models [82-84].

In the mean-field models of the VUU/BUU type the nucleons follow a trajectory which is generated by the en- 
semble averaged motion of all the other nucleons. This means that two-body and higher-order correlations are ignored, and these models give only event-averaged answers for many-body observables such as the formation of fragments [52,59].

This limitation can be overcome with the molecular dynamics models, where the nucleons are propagated with respect to the explicit interactions with all the other nucleons. Thus, these models can also treat the multifragmentation of the system. However, the classical molecular dynamics models neglect all quantum effects, which are most important in heavy ion collisions (see Ref. [74]), i.e., Pauli blocking, stochastic scattering, and particle production. In order to overcome these limitations the quantum molecular dynamics (QMD) model has been developed [70-77], which incorporates the quantum effects of the VUU and cascade models into the molecular dynamics approach.

A general deficiency of all microscopic models in the past was that the nuclear ground state could not be simulated in a reasonable fashion, because of the absence of the Fermi motion generated by the Pauli exclusion principle. Wilets et al. [62,63] suggested that the Pauli exclusion principle might be simulated by a momentumdependent, repulsive potential and it was demonstrated recently that it is in fact possible to design Pauli potentials so that the ensuing quasiclassical models give a reasonable reproduction of gross thermostatic properties of a Fermi gas and of nuclear matter [81,85]. It was shown [85] that the nuclear equation of state at subsaturation densities is strongly modified due to the clustering of nuclear matter.

For the description of the dynamical process of multifragmentation in heavy ion collisions we suggest a two step model, where the first step treats the nonequilibrium part of the reaction in a microscopic way. This step also describes the formation of highly-excited fragments. The decay of these fragments is further described with a different model (i.e., a statistical model). In fact, the time scale of the two types of processes necessitates such a treatment: While the nonequilibrium reactions occur during typical collision times of the order $10^{-22} \mathrm{sec}$, the evaporation and fission decay of the fragments may happen after relatively long times of the order $10^{-21}-10^{-15} \mathrm{sec}$. It is clear, even though in principle possible, that such long time behavior cannot be followed explicitly on the computer.

We discuss the first stage of this model in the present work. We incorporate the Pauli potential of Ref. [85] into the quantum molecular dynamics model [70-77] and show some properties of results calculated with this modified approach. In Sec. II we give a short description of the model. We show how the thermostatic properties of the Fermi gas, as well as the static properties of finite nuclei, can be reproduced. In Sec. III we investigate the decay of a hot nucleus and of compressed nuclei within the framework of the QMD model. In Sec. IV we compare preequilibrium neutron spectra obtained with this model with experimental data in order to illustrate the success of the dynamics of the model for (nucleon in, nucleon out) reactions. Conclusions are given in Sec. V.

\section{THE MICROSCOPIC DESCRIPTION OF FERMIONIC SYSTEMS}

\section{A. Description of free fermions}

In the present work we employ the Gaussian Pauli potential introduced by Dorso et al. [81]. With such a potential the total energy of the "free" Fermi gas is given by

$$
E_{\mathrm{tot}}^{\mathrm{FG}}=E_{\mathrm{kin}}+E_{\mathrm{Pau}}=\sum_{i} \frac{\mathbf{p}_{i}^{2}}{2 m}+\frac{1}{2} \sum_{i \neq j} V_{\mathrm{Pau}}^{0}\left[\frac{\hbar}{q_{0} p_{0}}\right]^{2} \exp \left[-\frac{r_{i j}^{2}}{2 q_{0}^{2}}-\frac{p_{i j}^{2}}{2 p_{0}^{2}}\right] \delta_{\tau_{i} \tau_{j}} \delta_{\sigma_{i} \sigma_{j}},
$$

where $\tau_{i}$ and $\sigma_{i}$ denote the spin-isospin index of nucleon $i$.

In Ref. [85] we parametrized this Pauli potential such that the total energy $E_{\text {tot }}^{\mathrm{FG}}$ matches the exact kinetic energy of the Fermi gas. This parametrization, however, may influence the dynamical calculations, since the kinetic part of the Fermi motion is reduced, which crucially influences, for example, the nucleon-nucleon collisions and therefore also the thermalization process. For this reason we also employ a second parametrization, where the kinetic energy alone compares to the exact kinetic energy of the Fermi gas. We denote these parameter sets from now on with 1 and 2, respectively (see Table I).

First we present the calculated thermostatic properties of an infinite system of free Fermions calculated within the framework presented. To do so we treat 512 nucleons (128 for each spin-isospin degree of freedom) in a cube with periodic boundary conditions. The thermostatic properties are obtained by averaging over several hundred statistically distributed manifestations of the system, sampled by means of the Metropolis importance sampling method [86] on the basis of the appropriate weight

$$
w=e^{-E_{\mathrm{tot}}^{\mathrm{FG}} / T} \text {. }
$$

Figure 1 shows the calculated energy per nucleon for

TABLE I. Parameters of the model for the different interactions.

\begin{tabular}{lcc}
\hline$K(\mathrm{MeV})$ & 1 & 2 \\
\hline$V_{\text {Pau }}^{0}(\mathrm{MeV})$ & 29 & 99.5 \\
$p_{0}(\mathrm{MeV} c)$ & 120 & 120 \\
$q_{0}(\mathrm{fm})$ & 3 & 3 \\
$L\left(\mathrm{fm}^{2}\right)$ & 2.1625 & 2.1625 \\
$\alpha(\mathrm{MeV})$ & -35.74 & -84.56 \\
$\beta(\mathrm{MeV})$ & 79.42 & 188.18 \\
$\gamma$ & 1.91 & 1.457 \\
$V_{\mathrm{Yuk}}^{0}(\mathrm{MeV})$ & -28 & -85 \\
$\gamma_{\mathrm{Yuk}}(\mathrm{fm})$ & $1 / 0.78$ & 1.0 \\
$\Omega(\mathrm{MeV})$ & 25 & 25 \\
\hline \hline
\end{tabular}


the two parameter sets for different temperatures versus the density of the system. For comparison, the solid lines show the corresponding exact values for a free Fermi gas. All three figures show the total energies and the kinetic energies for the two parameter sets for $T=0.5,5.0$, and $20.0 \mathrm{MeV}$. It can be seen in all cases that the total energy for set 1 and the kinetic energy for set 2 match the exact Fermi gas values. The Pauli potential is most important at low densities and temperatures and can be neglected in the Boltzmann limit. Figure 2 shows the corresponding results for different densities versus temperature.

The main effect that has to be reproduced for the present purpose is the value of the kinetic energy. This is achieved rather satisfactorily in all cases. In particular the ground state configuration no longer collapses in the limit $T \rightarrow 0$. However, the specific heat, i.e., the slope of the energy versus the temperature curves in Fig. 2, also can be reproduced. Figure 3 shows the specific heat at constant volume $c_{V}$ for the calculations with the two pa-

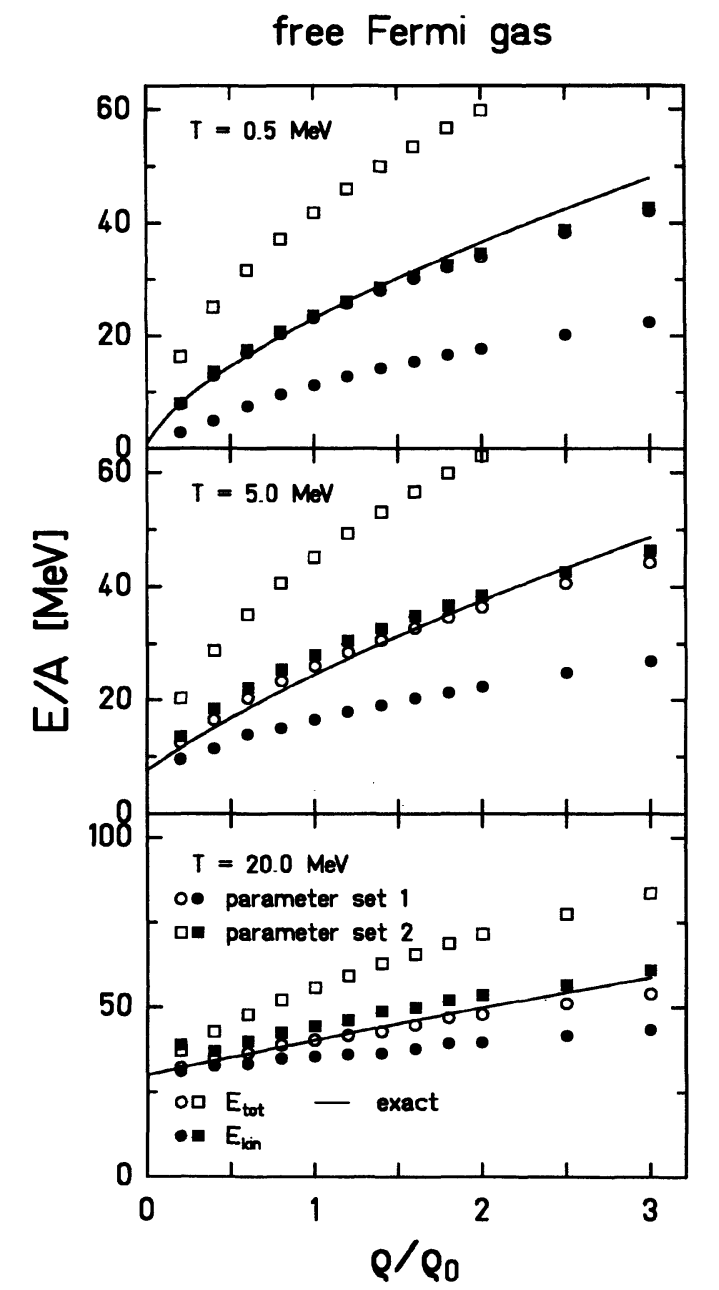

FIG. 1. The exact results for the energy per nucleon in a free Fermi gas is shown by the solid lines, while the symbols indicate the results calculated with the Metropolis algorithm using parametrizations 1 and 2 for different temperatures as indicated. Shown are both the total energy (open symbols) and the kinetic part of the energies (full symbols).

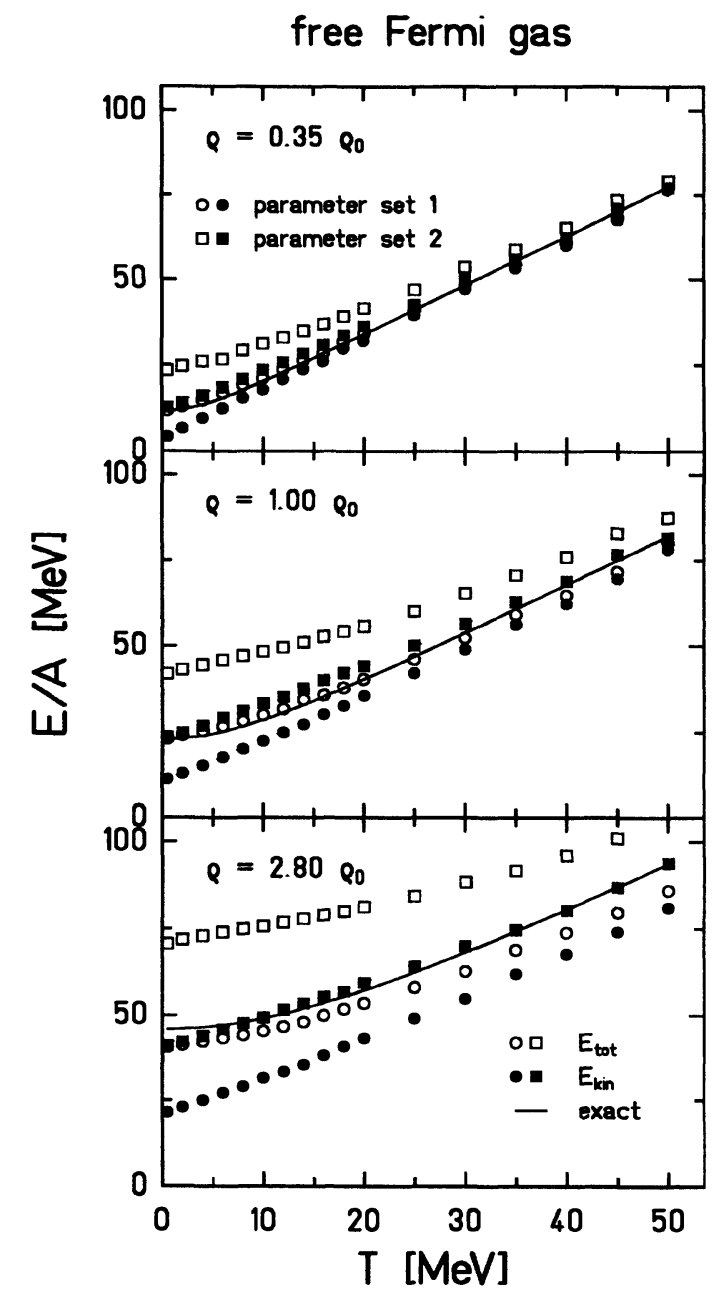

FIG. 2. Same as Fig. 1 for different densities versus temperatures.

rameter sets as compared to the exact value for the Fermi gas at $\rho=\rho_{0} . \quad c_{V}$ has been computed as $c_{V}=\Delta E_{\text {tot }} / \Delta T$ for parameter set 1 while the kinetic energy has been used for parameter set 2. The most important feature of the specific heat, namely, the drop from the Boltzmann value

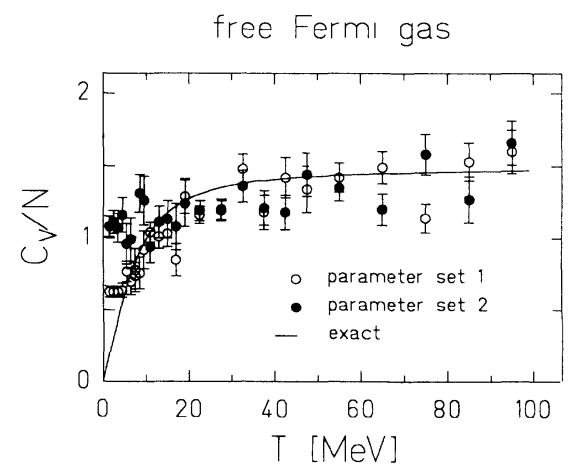

FIG. 3. Specific heat $c_{V}$ for the free Fermi gas, calculated at $\rho=\rho_{0}$ with the two parameter sets as indicated, compared to the exact values. 
$c_{V}=\frac{3}{2}$ to zero at low temperatures, is not correctly reproduced in both cases. The calculated curves do not approach zero for $T \rightarrow 0$; i.e., the Pauli potential lacks a quasiparticle limit. As can be seen from Fig. 3 both parameter sets fail to reproduce the specific heat for temperatures $T<5 \mathrm{MeV}$. The overall agreement with the exact values is better for parameter set 1 , while for parameter set $2 c_{V}$ decreases only weakly with decreasing temperature.

\section{B. Description of finite nuclei}

After this brief demonstration of the significant qualitative improvements effected by the Pauli potential, we describe the remainder of the many-body Hamiltonian. It is taken from the QMD model [70-77] and so it includes a two- and three-body Skyrme interaction as well as Coulomb, Yukawa, and asymmetry potentials.

The nucleons are represented by Gaussians of the form

$$
\begin{aligned}
f_{i}\left(\mathbf{r}_{i}, \mathbf{p}_{i}, t\right)=\frac{1}{(\pi \hbar)^{3}} \exp \{ & -\frac{\left[\mathbf{r}_{i}-\mathbf{r}_{i 0}(t)\right]^{2}}{2 L} \\
& \left.-\left[\mathbf{p}_{i}-\mathbf{p}_{i 0}(t)\right]^{2} \frac{2 L}{\hbar^{2}}\right\},
\end{aligned}
$$

where $\mathbf{r}_{i 0}$ and $\mathbf{p}_{i 0}$ are the centroids of particle $i$. Integration over momentum space yields the real-space distribution

$$
\tilde{f}_{i}\left(\mathbf{r}_{i}, t\right)=\frac{1}{(2 \pi L)^{3 / 2}} \exp \left\{-\frac{\left[\mathbf{r}_{i}-\mathbf{r}_{i 0}(t)\right]^{2}}{2 L}\right\}
$$

and the baryon density

$$
\rho_{B}(\mathbf{r}, t)=\sum_{i=1}^{N} \widetilde{f}_{i}(\mathbf{r}, t) .
$$

The Hamilton function must consist of the kinetic energy of the particles and the Pauli potential energies. In addition, the Yukawa, Coulomb, and Skyrme interactions have to be incorporated. This is done in the following way: With the Gaussian distributed nucleons [Eq. (3)] one calculates

$$
\begin{aligned}
H= & \left.\sum_{i=1}^{N}\left[\left(\mathbf{p}_{i 0}^{2}+m^{2}\right)^{1 / 2}-m\right]+\frac{1}{2} \sum_{\substack{i, j=1 \\
j \neq i}}^{N} V_{\mathrm{Pau}}^{0} \mid \frac{\hbar}{q_{0} p_{0}}\right]^{2} \exp \left[-\frac{\left(\mathbf{r}_{i 0}-\mathbf{r}_{j 0}\right)^{2}}{2 q_{0}^{2}}-\frac{\left(\mathbf{p}_{i 0}-\mathbf{p}_{j 0}\right)^{2}}{2 p_{0}^{2}}\right] \delta_{\tau_{i} \tau_{j}} \delta_{\sigma_{i} \sigma_{j}} \\
& +\frac{1}{2} \sum_{i, j=1}^{N} \int f_{i}\left(\mathbf{r}_{i}, \mathbf{p}_{i}, t\right) V_{\mathrm{Yuk}}^{0} \frac{e^{-\left|\mathbf{r}_{i}-\mathbf{r}_{j}\right| / \gamma_{\mathrm{Yuk}}}}{\left|\mathbf{r}_{i}-\mathbf{r}_{j}\right|} f_{j}\left(\mathbf{r}_{j}, \mathbf{p}_{j}, t\right) d \mathbf{r}_{i} d \mathbf{r}_{j} d \mathbf{p}_{k} d \mathbf{p}_{j} \\
& +\frac{1}{2} \sum_{i, j=1}^{N} \int f_{i}\left(\mathbf{r}_{i}, \mathbf{p}_{i}, t\right) \frac{e^{2}}{\left|\mathbf{r}_{i}-\mathbf{r}_{j}\right|} f_{j}\left(\mathbf{r}_{j}, \mathbf{p}_{j}, t\right) d \mathbf{r}_{i} d \mathbf{r}_{j} d \mathbf{p}_{i} d \mathbf{p}_{j} \frac{1}{4}\left(\sigma_{i}+1\right)\left(\sigma_{j}+1\right) \\
& +\frac{1}{2} \sum_{i, j=1}^{N} \int f_{i}\left(\mathbf{r}_{i}, \mathbf{p}_{i}, t\right) \alpha^{\prime} \delta\left(\mathbf{r}_{i}-\mathbf{r}_{j}\right) f_{j}\left(\mathbf{r}_{j}, \mathbf{p}_{j}, t\right) d \mathbf{r}_{i} d \mathbf{r}_{j} d \mathbf{p}_{i} d \mathbf{p}_{j} \\
& +\frac{1}{6} \sum_{\substack{j \neq i \\
j \neq i=1}}^{N} \int f_{i}\left(\mathbf{r}_{i}, \mathbf{p}_{i}, t\right) f_{j}\left(\mathbf{r}_{j}, \mathbf{p}_{j}, t\right) f_{k}\left(\mathbf{r}_{k}, \mathbf{p}_{k}, t\right) \beta^{\prime} \delta\left(\mathbf{r}_{i}-\mathbf{r}_{j}\right) \delta\left(\mathbf{r}_{i}-\mathbf{r}_{k}\right) d \mathbf{r}_{i} d \mathbf{r}_{j} d \mathbf{r}_{k} d \mathbf{p}_{i} d \mathbf{p}_{j} d \mathbf{p}_{k} \\
& +\frac{1}{2} \sum_{i, j=1}^{N} \int f_{i}\left(\mathbf{r}_{i}, \mathbf{p}_{i}, t\right) \Omega \sigma_{i} \sigma_{j} \delta\left(\mathbf{r}_{i}-\mathbf{r}_{j}\right) f_{j}\left(\mathbf{r}_{j}, \mathbf{p}_{j}, t\right) d \mathbf{r}_{i} d \mathbf{r}_{j} d \mathbf{p}_{i} d \mathbf{p}_{j} .
\end{aligned}
$$

Here $\sigma_{i}$ is 1 for protons and -1 for neutrons. Evaluating the integrals yields

$$
\begin{array}{r}
H=\sum_{i=1}^{N}\left[\left(\mathbf{p}_{i 0}^{2}+m^{2}\right)^{1 / 2}-m\right]+\frac{1}{2} \sum_{\substack{i, j=1 \\
j \neq i}}^{N} V_{\mathrm{Pau}}^{0}\left[\frac{\hbar}{q_{0} p_{0}}\right]^{2} \exp \left[-\frac{\left(\mathbf{r}_{i 0}-\mathbf{r}_{j 0}\right)^{2}}{2 q_{0}^{2}}-\frac{\left(\mathbf{p}_{i 0}-\mathbf{p}_{j 0}\right)^{2}}{2 p_{0}^{2}}\right] \delta_{\tau_{i} \tau_{j}} \delta_{\sigma_{i} \sigma_{j}} \\
+\frac{1}{2} \sum_{\substack{i, j=1 \\
j \neq i}}^{N} \frac{V_{\mathrm{Yuk}}^{0}}{2\left|\mathbf{r}_{i 0}-\mathbf{r}_{j 0}\right|} e^{L / \gamma_{\mathrm{Yuk}}^{2}}\left\{e^{-\left|\mathbf{r}_{i 0}-\mathbf{r}_{j 0}\right| / \gamma_{\mathrm{Yuk}}}\left[1-\operatorname{erf}\left[\frac{2 L / \gamma_{\mathrm{Yuk}}-\left|\mathbf{r}_{i 0}-\mathbf{r}_{j 0}\right|}{\sqrt{4 L}}\right]\right]\right. \\
\left.-e^{+\left|\mathbf{r}_{i 0}-\mathbf{r}_{j 0}\right| / \gamma_{\mathrm{Yuk}}}\left[1-\operatorname{erf}\left[\frac{2 L / \gamma_{\mathrm{Yuk}}+\left|\mathbf{r}_{i 0}-\mathbf{r}_{j 0}\right|}{\sqrt{4 L}}\right]\right)\right\}
\end{array}
$$




$$
\begin{aligned}
& +\frac{1}{2} \sum_{\substack{i, j=1 \\
j \neq i}}^{N} \frac{e^{2}}{\left|\mathbf{r}_{i 0}-\mathbf{r}_{j 0}\right|} \operatorname{erf}\left(\frac{\left|\mathbf{r}_{i 0}-\mathbf{r}_{j 0}\right|}{\sqrt{4 L}}\right) \frac{1}{4}\left(\sigma_{i}+1\right)\left(\sigma_{j}+1\right) \\
& +\sum_{i=1}^{N} \underbrace{\left.\frac{\alpha}{2} \frac{1}{(4 \pi L)^{3 / 2} \rho_{0}} \sum_{\substack{j=1 \\
j}}^{e^{-\left(\mathbf{r}_{i 0}-\mathbf{r}_{j 0}\right)^{2} / 4 L}+\frac{\beta}{\gamma+1}}\left[\frac{1}{(4 \pi L)^{3 / 2} \rho_{0}} \sum_{\substack{j=1 \\
j \neq i}}^{N} e^{-\left(\mathbf{r}_{i 0}-\mathbf{r}_{j 0}\right)^{2} / 4 L}\right]^{\gamma}\right]}_{\substack{j \neq i \\
j \neq i}}] \\
& +\sum_{i=1}^{N} \frac{\Omega}{2} \frac{1}{(4 \pi L)^{3 / 2} \rho_{0}} \sum_{\substack{j=1 \\
j \neq i}}^{N} \sigma_{i} \sigma_{j} e^{-\left(\mathbf{r}_{i 0}-\mathbf{r}_{j 0}\right)^{2} / 4 L}
\end{aligned}
$$

The first term denotes the kinetic energy of the centroids of the nucleons; the second term describes the Pauli potential, which acts only on the centroids of the Gaussians. The third and fourth terms are the Yukawa and Coulomb interactions, respectively, for Gaussian-shaped wave packets representing the nucleons. The last term represents the Skyrme part of the interaction which can be considered as a density-dependent interaction for infinite nuclear matter. Note, however, the difference between $\rho_{i}^{\prime}$ in Eq. (7) and $\rho_{B}$ in Eq. (5). Those two terms are equal only for infinite systems.

The parameters $\alpha, \beta$, and $\gamma$ are adjusted so as to yield reasonable values for the binding energy, saturation density, and compressibility of infinite nuclear matter. For the present study we use a compressibility constant of $K=380 \mathrm{MeV}$. Note, however, that the equation of state is substantially softened by the clustering of nuclear matter at subsaturation densities [85].

In order to obtain a reasonable simulation of finite nuclei, we adjust the two Yukawa parameters $V_{\mathrm{Yuk}}$ and $\gamma_{\text {Yuk }}$. The Yukawa potential also gives a densitydependent contribution to the equation of state, which must be taken into account when adjusting the parameters. The resulting parameters are given in Table I.

Now we present the thermostatic properties of cold nuclei using this Hamiltonian in the weight factor [Eq. (2)] of the Metropolis sampling algorithm. We use the Metropolis algorithm to obtain an ensemble average of self-bound clusters at the temperature $T=0.5 \mathrm{MeV}$,

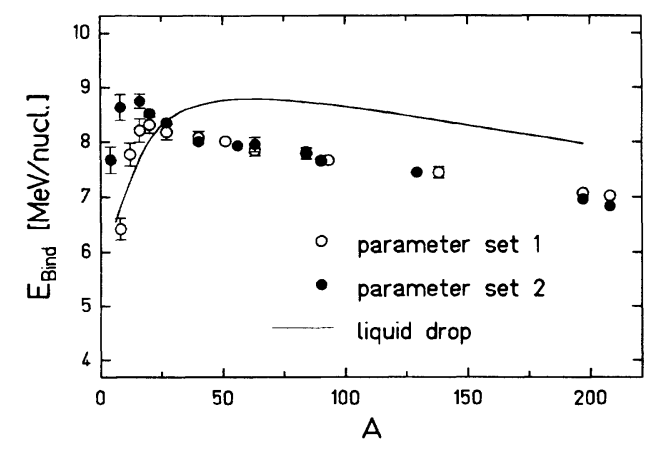

FIG. 4. Energy per nucleon of the calculated nuclei (at $T=0.5 \mathrm{MeV}$ ) with the parametrizations as indicated, compared to the Bethe-Weizsäcker values. which is a sufficiently good approximation to cold nuclei in the present context.

Figure 4 shows the binding energies of nuclei calculated with the two parameter sets compared to the BetheWeizsäcker values (full lines); Fig. 5 compares the rootmean-square radii. Both parameter sets reasonably reproduce the radii and give values of $\sim 7-8.5$ $\mathrm{MeV} /$ nucleon for the binding energies, but they favor light nuclei in the binding energy curve. The reason for this is that the microscopic Skyrme forces, which give the density-dependent repulsion, cannot compensate the strong Yukawa interaction, which is necessary to get the proper surface binding energy for large nuclei. The Pauli potential acts explicitly between spin-isospin identical particles. This may give a poor description of the Fermi energy in very light nuclei. This is also the reason why the Yukawa parameters in Table I deviate from the standard values.

The binding energy of heavy nuclei is about 1 $\mathrm{MeV} /$ nucleon too low, and the maximum of the curve is shifted to lighter nuclei than predicted by the BetheWeizsäcker mass formula. Within the present microscopic model this is the best parametrization we could find. Note also that these nuclei were constructed in a canonical manner within a heat bath of $0.5 \mathrm{MeV}$ temperature. If we use a downhill simplex method to construct the ground state for $T=0 \mathrm{MeV}$, we find that the excitation energy corresponding to the temperature of $0.5 \mathrm{MeV}$ is about 0.5 to $1.0 \mathrm{MeV} /$ nucleon for heavy nuclei [75].

Figure 6 shows the corresponding density profiles for three typical ground state nuclei. The densities have been obtained using Eq. (5). The error bars denote the statisti-

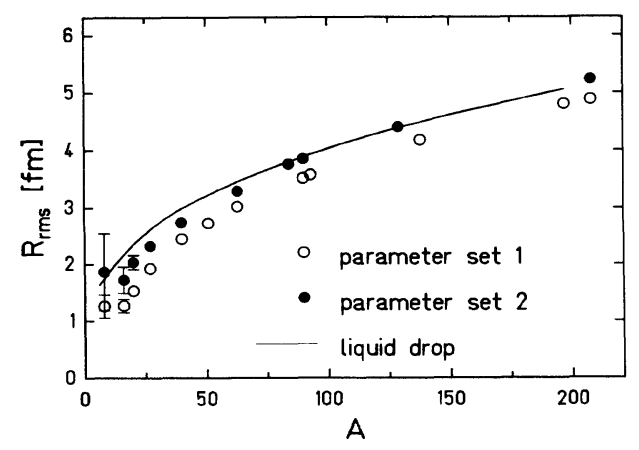

FIG. 5. Same as Fig. 4 for the root-mean-square radii. 


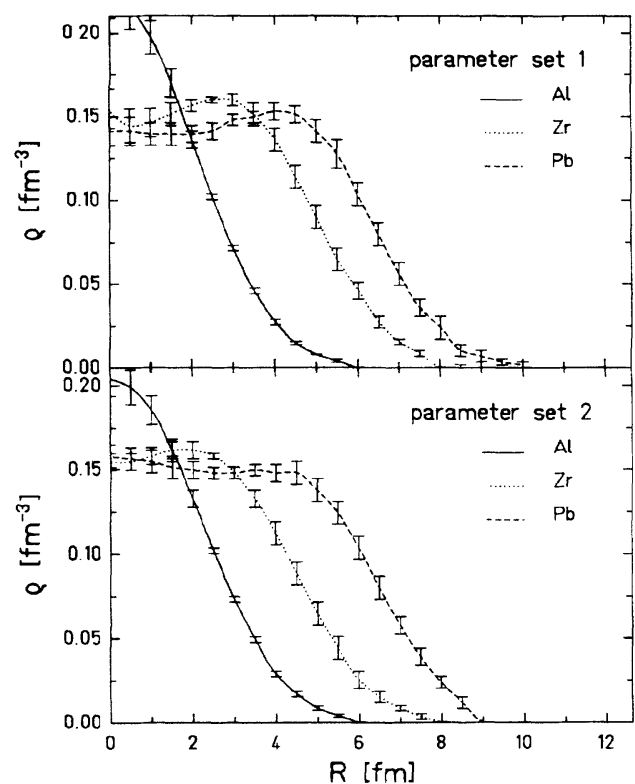

FIG. 6. Density profiles for some ground state nuclei.

cal errors within an ensemble of 10 events for each nucleus. The small values of the error bars indicate that this ground state is reproduced not only on the average but for each event. The shape of the density distributions show a central density of $\approx 0.15-0.16 \mathrm{fm}^{-3}$ for heavy nuclei while the lighter ones (e.g., Al) are peaked at the center. The value of the central density is determined by the parametrization of the Skyrme forces while the surface thickness is determined by the width of the Gaussian shaped nucleons [see Eq. (3)] and the parameters of the Yukawa potential.

\section{Dynamical stability of nuclei}

Until now we have presented only thermostatic nuclear properties obtained with the Metropolis algorithm. Now we present results of dynamical calculations within the quantum molecular dynamics model described in Refs. [70-77]. Since we are now dealing with momentumdependent interactions the leapfrog integration routine used previously is replaced by a second-order RungeKutta method. In addition to that, the initialization of projectile and target is performed with the Metropolis procedure described above, rather than by using a local Thomas-Fermi approach as in previous versions of this model.

First we want to study the dynamical stability of the nuclei. We prepare the initial nuclei using the Metropolis sampling procedure at $T=0.5 \mathrm{MeV}$ and take this state as the starting point for the dynamical QMD calculation.

Figure 7 shows the time evolution of the root-meansquare radii for three different nuclei $\left({ }^{20} \mathrm{Ne},{ }^{100} \mathrm{Ru}\right.$, and $\left.{ }^{197} \mathrm{Au}\right)$ calculated with the two parameter sets. Since we want to show the fluctuations here, we present only one event for each case. We observe that those nuclei represent a good approximation to the real ground state

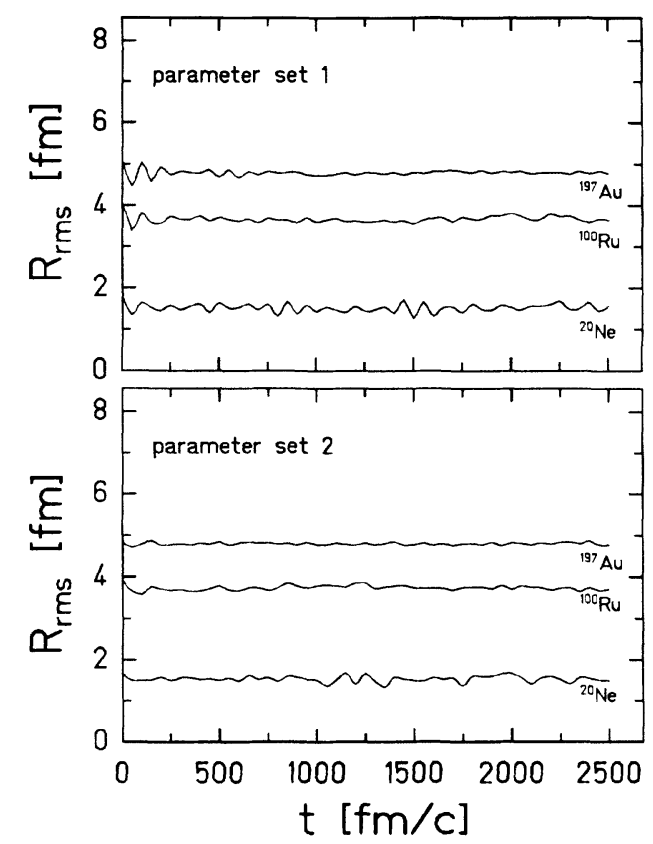

FIG. 7. Stability of ground state nuclei computed with the two parameter sets.

and are therefore stable for very long time scales. Both parameter sets yield equally stable nuclei. Therefore we conclude that this stability results not from the fact that the kinetic energies are artificially reduced (with parameter set 1), since the same stability is also found with parameter set 2 . The small oscillations are due to the fact that these nuclei still have a small excitation energy corresponding to $0.5 \mathrm{MeV}$ temperature.

\section{Determination of the excitation energy of the fragments}

Finally we want to describe the calculation of the excitation energies of the emitted fragments. We define the fragments at the end of the reaction using a common minimum spanning tree procedure. Two nucleons are considered to be bound in a fragment if the centroids of their wave packets have a spatial distance $d_{0} \leq 3 \mathrm{fm}$. Then we determine the total energy $E^{1}$ of each fragment in its rest frame and use the Metropolis procedure to evaluate the ground state (with the energy $E^{0}$ ) for each particular fragment. The difference of these energies gives us the excitation energy of each fragment on an event-by-event basis,

$$
E^{*}=E^{1}-E^{0} \text {. }
$$

It should be noted that this procedure gives the energy with an uncertainty of about $1 \mathrm{MeV} /$ nucleon (see also the discrepancy of the binding energies compared to the experimental values in Fig. 4). This may be relevant for further applications of these excitation energies (as input to the second step of a two-step model). The sensitivity of the results to this uncertainty has been investigated [76] and it was found that, e.g., the change of the multiplicities of intermediate mass fragments in central heavy ion collisions, due to this uncertainty, is less than $20 \%$. 


\section{DEEXCITATION OF EXCITED NUCLEI}

In this section we investigate how our model describes the deexcitation of highly excited, i.e., hot or compressed, nuclei. From the results of the statistical models [40-49], we know that a hot nucleus can decay into more than one large $(A>4)$ fragments (this process is called "multifragmentation"), when a critical temperature of about $4 \mathrm{MeV}$ is exceeded. The same process is supposed to occur when a compressed nucleus starts to expand and crosses the boundary line between the nuclear liquid and vapor phase. While the first process plays an important role in high-energy proton-induced reactions, where the target is highly excited without being compressed, the second process should play an important role in heavy ion reactions, since the projectile-target system may be heated up and compressed and may then freely expand. However, it is certainly not clear whether a multifragmentation event in those two processes results from the same physical origin. As a test, we wish to see if our dynamical model, if started in an excited, equilibrated initial condition will reproduce the results of a statistical model calculation.

To do so we prepare the nuclei at some distinct temperature and compression energy using the Metropolis algorithm. Since this algorithm uses a canonical description, we put an infinitely high potential well at the radius $R=R_{0}$ and do the canonical Metropolis sampling until we reach a global equilibrium inside this well. Then we remove the well and start the microcanonical molecular dynamics calculation. Since we do not know precisely what causes the decay and especially the multifragmentation of hot nuclei, we investigate this process both in a pure molecular dynamical (MD) calculation (without the hard $n-n$ collisions) and with the full QMD model including collisions.

We study the decay of a ${ }^{100} \mathrm{Ru}$ nucleus using the different parameter sets of our model (see Sec. II) and follow the time evolution for $2500 \mathrm{fm} / c$ using a secondorder Runge-Kutta integration routine with a timestep of $0.5 \mathrm{fm} / c$. First we prepare the nuclei at normal density $\left(R_{0}=1.13 A^{1 / 3} \mathrm{fm}-0.3 \mathrm{fm}\right)$ and vary the temperature between 0 and $20 \mathrm{MeV}$.

Figure 8 shows the multiplicities of five different fragment types ( $A=1,2-4,5-15,16-50,51-100)$ for the two parameter sets with (right column) and without (left column) the hard collisions versus the thermal excitation energy. Up to an excitation energy of $\approx 8-10$ $\mathrm{MeV} /$ nucleon one large $(A>50)$ residue survives. For higher energies these residues get smaller and populate the mass bins $A=16-50$ and $A=5-15$, but for all temperatures no multifragmentation $[\operatorname{Mul}(A>4) \approx 1$, in all cases] can be observed. The only deexcitation channel is the evaporation of single nucleons $(A=1)$ and few light fragments $(A=2-4)$. Obviously there is no significant difference between the two parametrizations or between the calculations with and without the collisions. This leads us to the conclusion that this decay process is neither influenced by the momentum distribution of the nucleons (the main difference between the two parametrizations is the distribution of the kinetic energies of the nu-

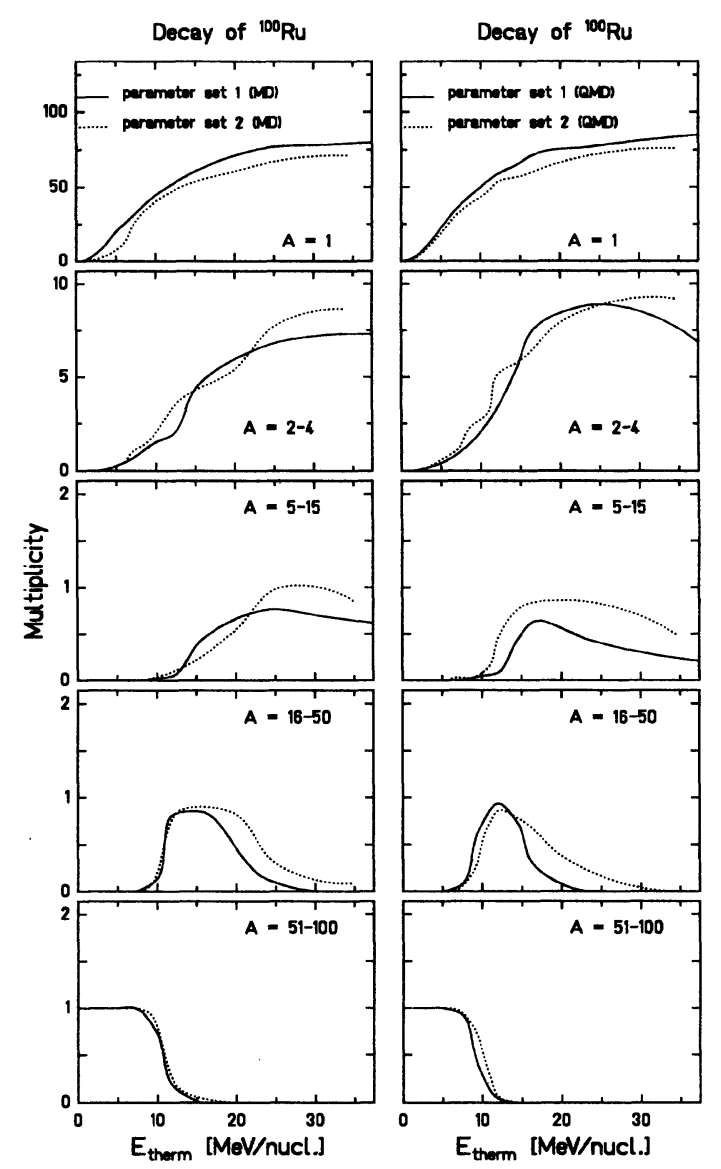

FIG. 8. The multiplicities of different fragments are shown for the deexcitation of an excited ${ }^{100} \mathrm{Ru}$ nucleus at normal density and different temperatures.

cleons as can be seen in Figs. 1 and 2) nor by nonequilibrium processes such as hard scattering. This behavior is in contrast to the statistical models of multifragmentation which predict much higher multiplicities of IMFs. Those models, however, do not consider the dynamical process of fragment formation but assume a fragmentation and calculate only the most probable fragment distribution via phase space arguments. The question whether the phase space alone determines the outcome of these reactions, or if dynamical constraints (as seen, for example, in nuclear fission) will restrict the multifragmentation channel, is still not answered. In our model the most probable deexcitation mechanism is the evaporation of nucleons from the high-energy tail of the Fermi distribution.

Now we switch over to the second case, where we start with a cold, compressed nucleus. This case is of academic interest, since it is not possible to compress a nucleus without heating it up in nature. An advantage of our model, however, is that those physical situations, which are mixed in nature, can be disentangled. This is achieved in this case by putting the potential well in the initialization at the radius $R=R^{\prime} R_{0}$ (with $R^{\prime}<1$ ). The Metropolis sampling is then done for the temperature $T=0.5 \mathrm{MeV}$ until a global equilibrium for this compressed but cold nucleus is achieved. Figure 9 shows 
the same multiplicity distributions as before versus the average excitation energy of the compressed nuclei. Now the large residue starts to decay at lower energies and a difference between the two parameter sets can be observed. The nucleus with the Pauli parameter set 2 seems to be more stable, which may result from the stronger attraction due to the Yukawa interaction, which also yields larger binding energies for small nuclei as can be seen in Fig. 4.

If the energy is increased then in all cases a clear multifragmentation into up to 6 large $(A>4)$ fragments results, which mostly populate the interval $A=5-15$, where the largest binding energy can be found in Fig. 4. The multifragmentation curve is peaked at $E^{*} \approx 10$ $\mathrm{MeV} /$ nucleon. Note that the same qualitative behavior was found in previous QMD calculations [71]. The fragment multiplicities in central $\mathrm{Nb}+\mathrm{Nb}$ collisions plotted versus the bombarding energy exhibit a peak at $E_{\text {beam }} \approx 100 \mathrm{MeV} /$ nucleon. Again we observe that the parameter set 2 yields fewer fragments.

Since these calculations are from a dynamic model, we can look at the time evolution of the deexcitation process. In Fig. 10 we show the average excitation energy [cf. Eq. (8)] of the heavy $(A>4)$ fragments versus reaction time.

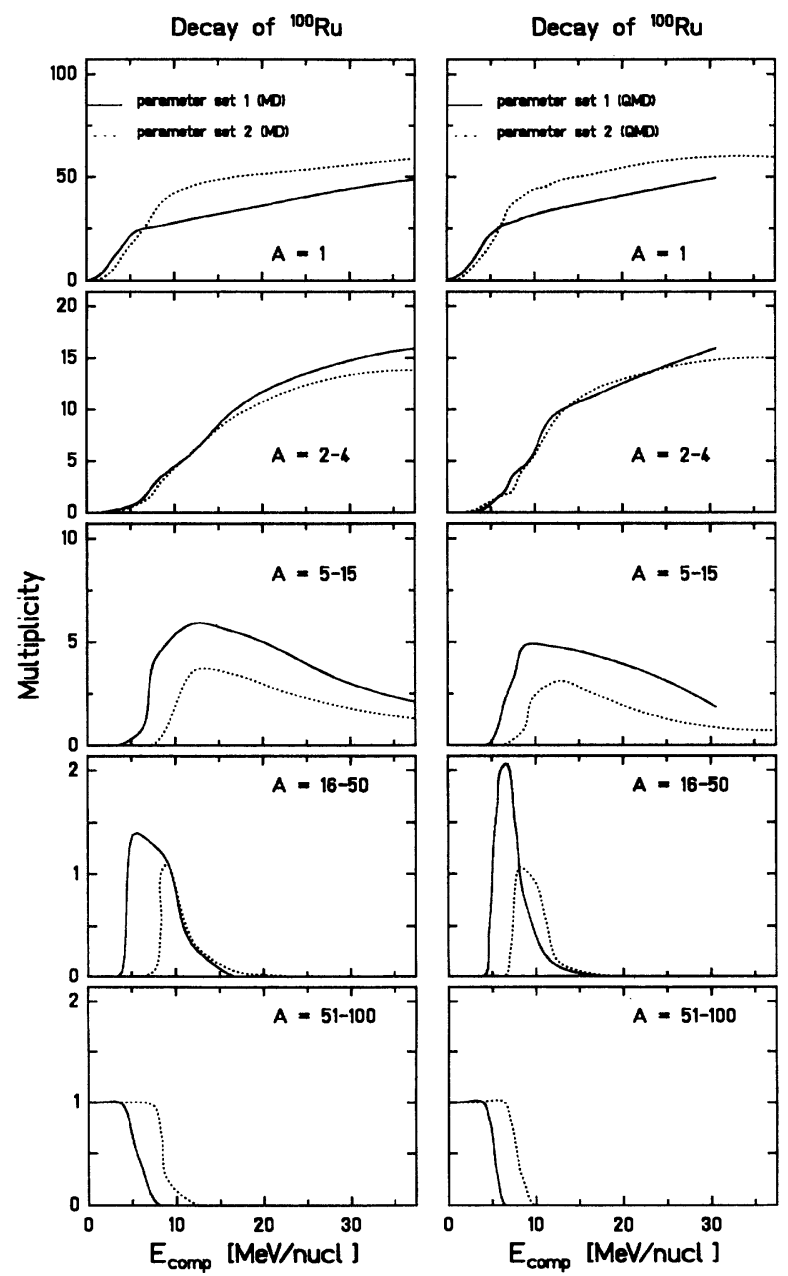

FIG. 9. Same as Fig. 8, but now with nuclei initialized and $T=0.5 \mathrm{MeV}$ at different densities. The abscissa shows the corresponding excitation energies per nucleon.

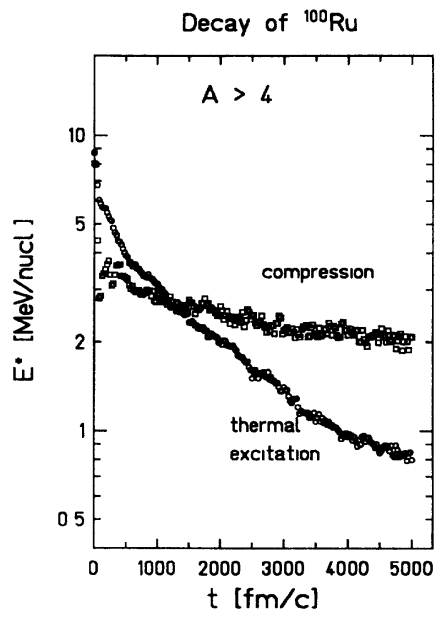

FIG. 10. Time evolution of the excitation energy of the remaining heavy fragments $(A>4)$ of Fig. 8 for an initially heated and an initially compressed nucleus.

Shown are the results for parameter set 1 at an initial excitation of $\approx 8 \mathrm{MeV} /$ nucleon for the two cases thermal and compressional excitation without collisions. The inclusion of the hard collisions, however, does not change this result. If the thermally excited nuclei are considered, the excitation energy decreases almost exponentially and reaches final values of about $1 \mathrm{MeV} /$ nucleon. In the case of the compressed but cold system a much faster "explosion" can be observed when the compression energy is transformed into collective translational motion. Within a very short timescale of about $50-100 \mathrm{fm} / c$ the excitation energy drops by $5 \mathrm{MeV} /$ nucleon. After this the further deexcitation also shows an exponential decrease of the excitation energy. Because of the smaller mass (in this case there are about five fragments in the mass regime $A=5-15$, while for the thermal case one large fragment with $A \approx 50$ was left) the further deexcitation is much slower, and therefore the remaining excitation energy after $5000 \mathrm{fm} / \mathrm{c}$ reaction time is about 1 $\mathrm{MeV} /$ nucleon higher than for the thermal case. This difference of the rest energy after $5000 \mathrm{fm} / c$ reaction time is due to the different timescales for the deexcitation of large (thermal excitation) and small (compression) fragments and does not result from any collective degrees of freedom such as rotation or vibration.

Thus the QMD model does not describe the multifragment decay of thermally excited nuclei, while for mechanically excited (compressed) nuclei the multifragmentation channel is predicted. On this basis one might conclude that, according to the QMD calculations, true multifragmentation processes are caused by a dynamical rupture of the system and not by "thermal boiling." It has to be seen, however, whether more refined evaporation models, which suppress the level densities of the clusters in the continuum, due to restriction to bound and quasibound levels [87], yield similar results.

\section{PREEQUILIBRIUM NEUTRON EMISSION}

Preequilibrium neutron emission has been measured recently for the inclusive reactions $p+\mathrm{Al}, \mathrm{Zr}, \mathrm{Pb}$ at 


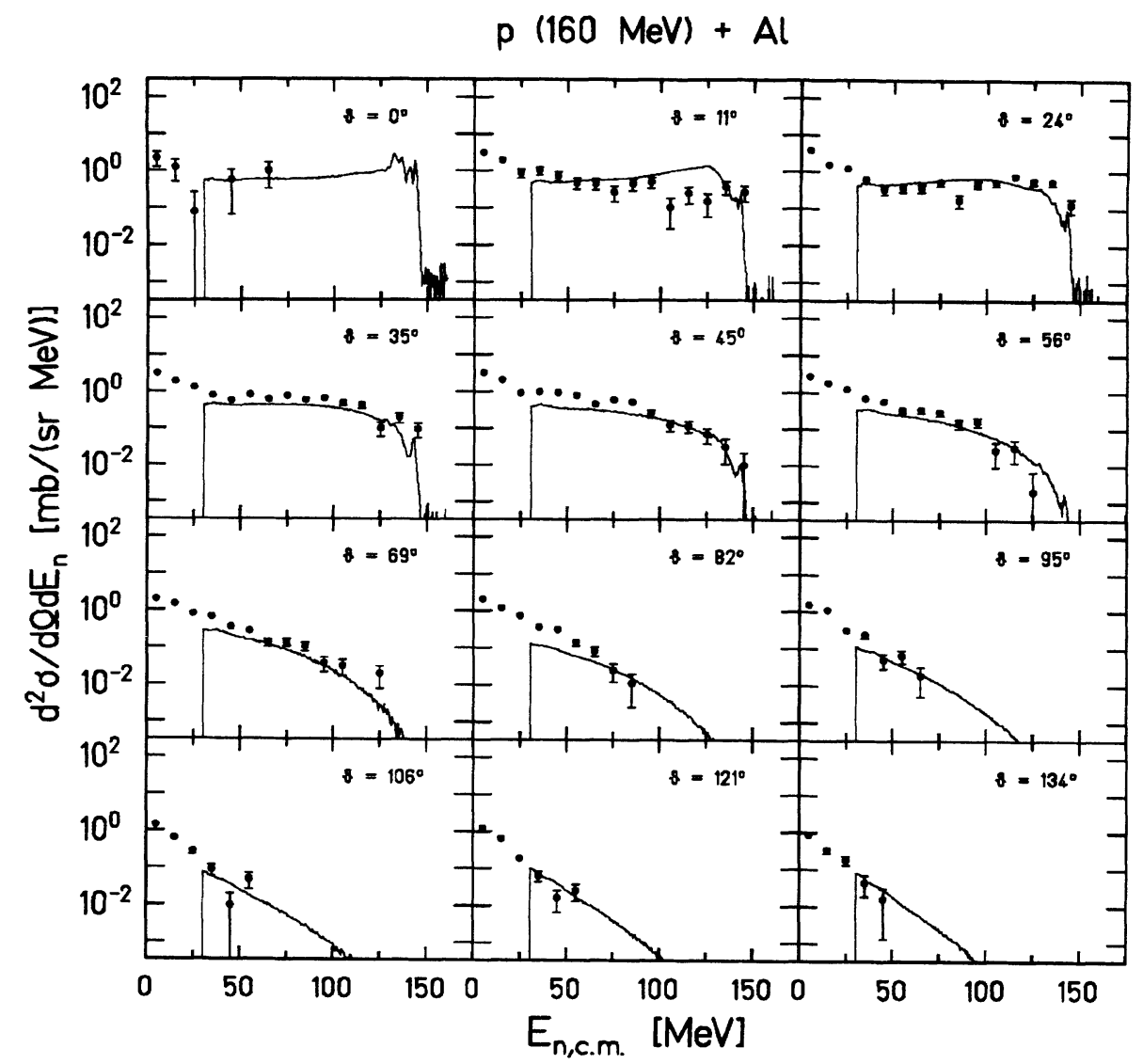

FIG. 11. Neutron energy spectra in the c.m. frame for the reaction $p(160 \mathrm{MeV})+\mathrm{Al}$ at different laboratory angles as indicated. The symbols show the QMD calculation while the full line represents the data of Ref. [94].

$E_{p}=80,120$, and $160 \mathrm{MeV}$ [94]. It has been reported [89-92] that in this energy regime semiclassical preequilibrium models, which are based on an intranuclear nucleon-nucleon collision process, fail to reproduce the angular distributions. In order to describe the data more appropriately a multistep model based on the FeshbachKerman-Koonin (FKK) [88] formalism with at least two or more incoherent direct nucleon-nucleon interactions has been used [94]. Since the QMD model follows the trajectories of all nucleons microscopically and treats both the hard nucleon-nucleon scattering and the soft nucleon-nucleon interaction, it should be equally successful in describing these reactions. In the QMD model, however, the soft nucleon-nucleon interaction is treated in a classical and not in a quantal way. The quantal as- pects of the scattering are taken into account at two points: (i) a collision is only allowed when the final scattering states are not Pauli blocked; (ii) the energy and angular dependence of the nucleon-nucleon cross section are taken from experiments. At energies $E<200 \mathrm{MeV}$ an isotropic, isospin-independent $40 \mathrm{mb}$ cross section is taken.

The experimental and calculated c.m. energy spectra of neutrons emitted at different laboratory angles are shown in Fig. 11 for the $\mathrm{Al}$ target at $160 \mathrm{MeV}$ incident proton energy, which is the highest energy measured until now; in Figs. 12-14 for the Zr target at 80, 120, and $160 \mathrm{MeV}$, respectively, and in Figs. $15-17$ for the $\mathrm{Pb}$ target at the same energies. Experiments at 256 and $800 \mathrm{MeV}$ incident energies will be performed to complete this data set.

TABLE II. Parametrizations of the neutron spectra for neutrons with $E_{n}=35 \mathrm{MeV}$.

\begin{tabular}{|c|c|c|c|c|c|c|}
\hline \multicolumn{7}{|c|}{$E_{p}(\mathrm{MeV})$} \\
\hline & & 80 & 120 & 160 & 256 & 800 \\
\hline \multirow[t]{2}{*}{ Al } & $A(\mathrm{mb} / \mathrm{MeV} \mathrm{sr})$ & 0.0722 & 0.1684 & 0.2710 & 0.1306 & 0.2063 \\
\hline & $B$ & 2.62 & 1.92 & 1.56 & 1.79 & 0.68 \\
\hline \multirow[t]{2}{*}{$\mathrm{Zr}$} & $A(\mathrm{mb} / \mathrm{MeV} \mathrm{sr})$ & 0.4226 & 0.5943 & & 0.6519 & 0.8552 \\
\hline & $B$ & 2.28 & 1.61 & & 1.16 & 0.14 \\
\hline \multirow[t]{2}{*}{$\mathrm{Pb}$} & $A(\mathrm{mb} / \mathrm{MeV} \mathrm{sr})$ & 0.7512 & 0.9532 & 1.1442 & 1.0213 & 1.6912 \\
\hline & $B$ & 1.59 & 1.68 & 1.76 & 1.33 & 0.57 \\
\hline
\end{tabular}




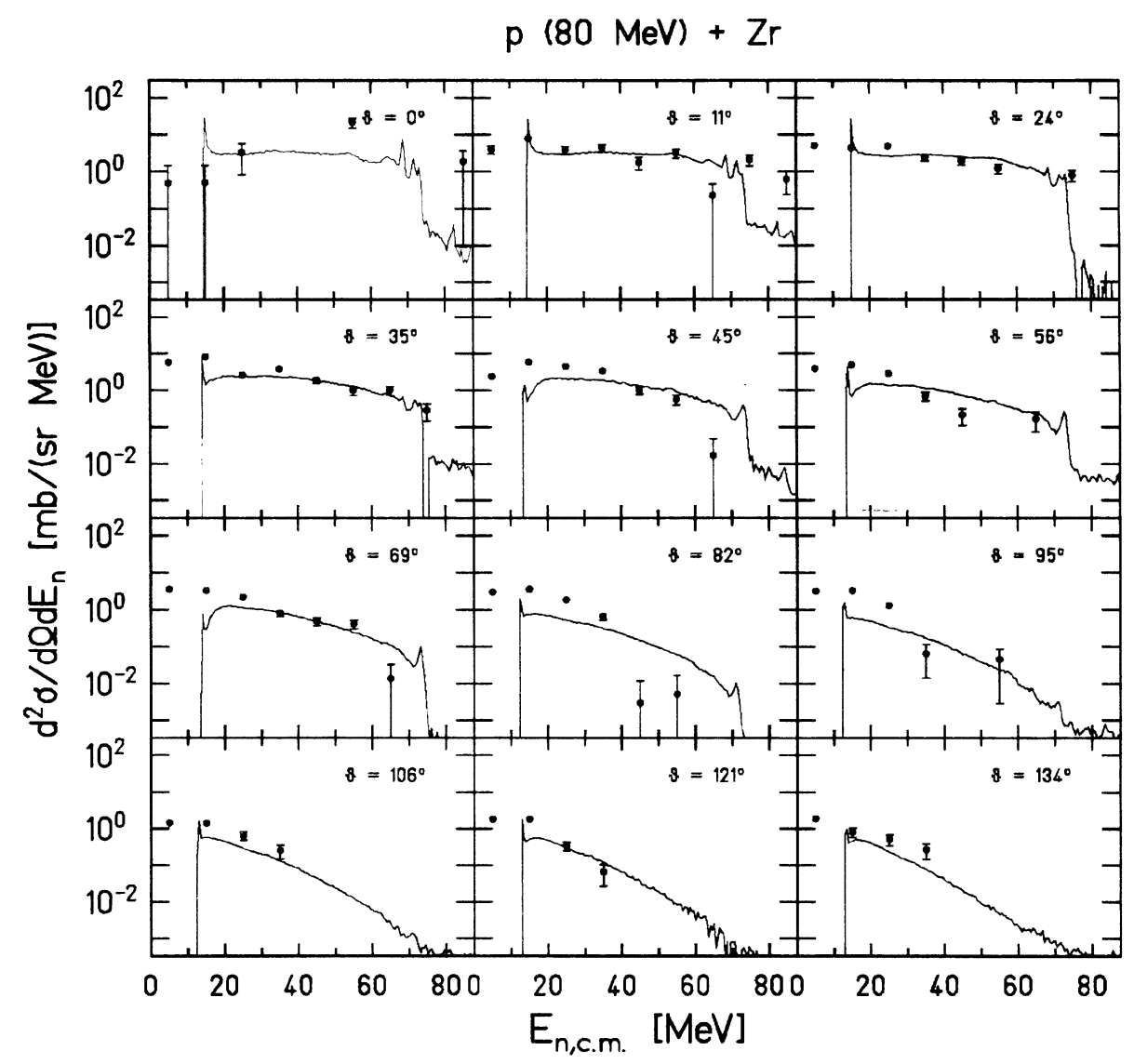

FIG. 12. Same as Fig. 11 for the reaction $p(80 \mathrm{MeV})+\mathrm{Zr}$.

The theoretical calculations have been performed using the full QMD (with collisions) and parameter set $1 .^{1}$ In all cases the calculated results agree with the data. Both the data and the calculations show a characteristic transition from a weak neutron energy dependence at forward angles to an almost exponential shape at backward angles. This clearly indicates that the system is not equilibrated. So we conclude - in agreement with [94] - that the failure of the previous preequilibrium models to describe the angular dependence of the data is due to the neglection of second- and higher-order collisions. The treatment of the collisions within the quantum mechanical model of Feshbach et al. gives a similar agreement with the data [94], which implies that the quantal treatment of the statistical multistep direct emissions is well reproduced with the stochastic collision term in the QMD model. Note also that this agreement was obtained using an isotropic nucleon-nucleon cross section. The detailed form of the nucleon-nucleon potential, which is the essential input, e.g., for the Feshbach-Kerman-Koonin theory, seems to be unimportant at these high energies.

Figures 18-20 show the angular distributions of neu-

TABLE III. Parametrizations of the neutron spectra for neutrons with $E_{n}=55 \mathrm{MeV}$.

\begin{tabular}{|c|c|c|c|c|c|c|}
\hline \multicolumn{7}{|c|}{$E_{p}(\mathrm{MeV})$} \\
\hline & & 80 & 120 & 160 & 256 & 800 \\
\hline \multirow[t]{2}{*}{$\mathrm{Al}$} & $A(\mathrm{mb} / \mathrm{MeV} \mathrm{sr})$ & 0.0048 & 0.0476 & 0.1101 & 0.0971 & 0.1336 \\
\hline & $B$ & 4.95 & 2.60 & 1.72 & 1.83 & 0.96 \\
\hline \multirow[t]{2}{*}{$\mathrm{Zr}$} & $A(\mathrm{mb} / \mathrm{MeV} \mathrm{sr})$ & 0.0283 & 0.2108 & 0.3934 & 0.2711 & 0.3838 \\
\hline & $B$ & 4.66 & 2.43 & 1.53 & 1.93 & 0.46 \\
\hline \multirow[t]{2}{*}{$\mathrm{Pb}$} & $A(\mathrm{mb} / \mathrm{MeV} \mathrm{sr})$ & 0.0632 & 0.3876 & 0.4189 & 0.6084 & 1.3653 \\
\hline & $B$ & 2.61 & 1.26 & 1.64 & 1.63 & 0.22 \\
\hline
\end{tabular}

${ }^{1}$ We also used parameter set 2 but could not find any significant differences. 


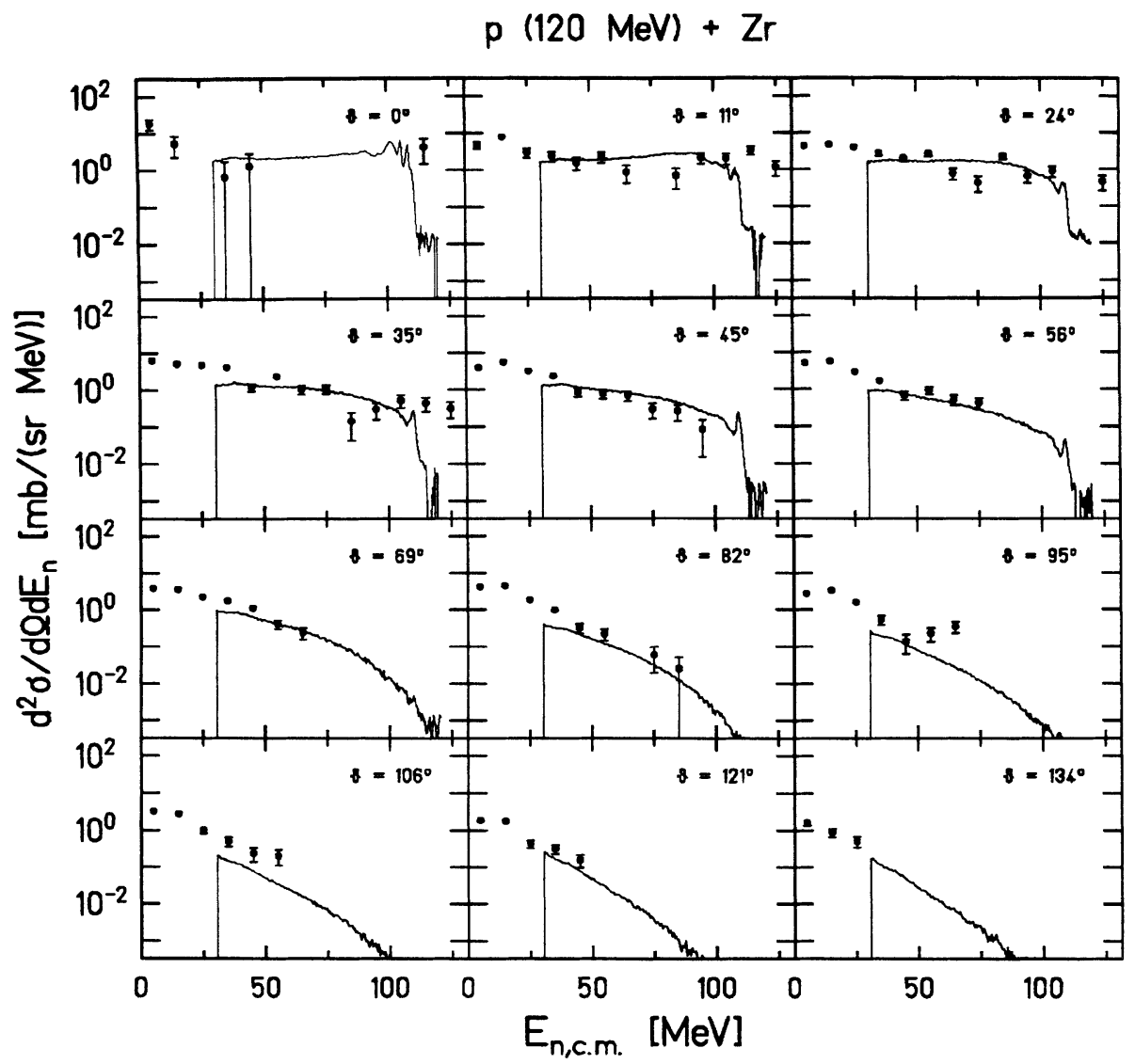

FIG. 13. Same as Fig. 11 for the reaction $p(120 \mathrm{MeV})+\mathrm{Zr}$.

trons with different c.m. energies. The data (open symbols) are compared with the QMD results (full symbols). The full lines show a parametrization of the QMD results in the form

$$
\frac{d^{2} \sigma}{d \Omega d E_{n}}=A \exp \left[B\left(E_{p}, E_{n}\right) \cos \theta\right]
$$

This parametrization has been proposed in Refs. $[93,94]$. Those authors claim that the slope parameter $B$ depends strongly on the neutron energies, and, for a given $E_{n}$, also on the bombarding energy but not on the target. In Tables II-IV we show the values of the parameters $A$ and $B$ for the curves in Figs. 18-20. (They have been obtained for neutrons with the indicated energy $\pm 10 \mathrm{MeV}$.) For the number of events run for these comparisons the QMD results give adequate statistics only for the low-energy neutrons.

Our calculations show, consistent with the data, that $B$ increases with increasing neutron energies (corresponding to steeper angular distributions). For a given neutron energy, $\boldsymbol{B}$ decreases with increasing bombarding energy. A significant dependence of the slope parameter on the mass of the target cannot be found within the statistical sampling error for the number of events we have considered thus far.

Tables V and VI show the parametrizations of the predicted neutron spectra for the high energy reactions at

TABLE IV. Parametrizations of the neutron spectra for neutrons with $E_{n}=75 \mathrm{MeV}$.

\begin{tabular}{|c|c|c|c|c|c|}
\hline \multicolumn{6}{|c|}{$E_{p}(\mathrm{MeV})$} \\
\hline & & 120 & 160 & 256 & 800 \\
\hline \multirow[t]{2}{*}{$\mathrm{Al}$} & $A(\mathrm{mb} / \mathrm{MeV} \mathrm{sr})$ & 0.0239 & 0.0114 & 0.0439 & 0.0939 \\
\hline & $B$ & 3.12 & 4.38 & 2.57 & 1.17 \\
\hline \multirow[t]{2}{*}{$\mathrm{Zr}$} & $A(\mathrm{mb} / \mathrm{MeV} \mathrm{sr})$ & 0.0082 & 0.2613 & 0.1930 & 0.3416 \\
\hline & $B$ & 5.46 & 1.89 & 2.28 & 0.77 \\
\hline \multirow[t]{2}{*}{$\mathrm{Pb}$} & $A(\mathrm{mb} / \mathrm{MeV} \mathrm{sr})$ & 0.1363 & 0.0512 & 0.5492 & 0.6912 \\
\hline & $B$ & 2.83 & 3.65 & 1.74 & 0.72 \\
\hline
\end{tabular}




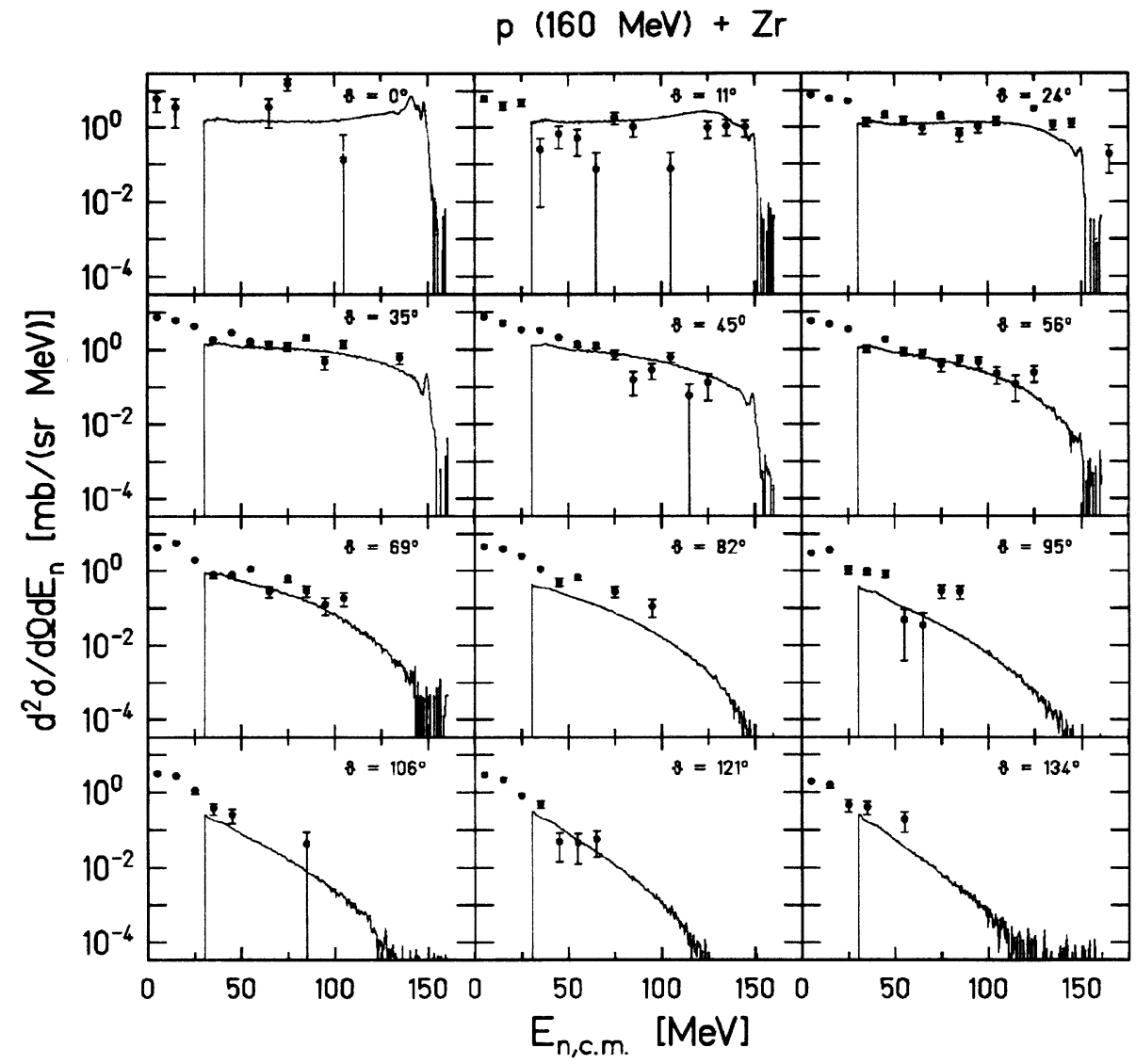

FIG. 14. Same as Fig. 11 for the reaction $p(160 \mathrm{MeV})+\mathrm{Zr}$.

256 and $800 \mathrm{MeV}$ incident energy. They confirm the trends that have been observed at low energies.

\section{CONCLUSIONS}

We have shown that the Pauli exclusion principle can be simulated in a quasiclassical way via a repulsive Pauli potential. Some of the thermodynamical properties of the free Fermi gas have been reproduced with a canonical

TABLE V. Parametrizations of the high-energy neutron spectra in the reactions $p(256 \mathrm{MeV})+\mathrm{Al}(\mathrm{Zr}, \mathrm{Pb})$.

\begin{tabular}{|c|c|c|c|c|}
\hline $\begin{array}{c}{ }_{E_{n}}^{p} \\
(\mathrm{MeV})\end{array}$ & $6 \mathrm{MeV})+$ & Al & $\mathrm{Zr}$ & $\mathrm{Pb}$ \\
\hline \multirow[t]{2}{*}{105} & $A(\mathrm{mb} / \mathrm{MeV} \mathrm{sr})$ & 0.0465 & 0.1443 & 0.3388 \\
\hline & $B$ & 2.62 & 2.30 & 2.00 \\
\hline \multirow[t]{2}{*}{125} & $A(\mathrm{mb} / \mathrm{MeV} \mathrm{sr})$ & 0.0143 & 0.0384 & 0.1765 \\
\hline & $B$ & 3.84 & 3.84 & 2.50 \\
\hline \multirow[t]{2}{*}{150} & $A(\mathrm{mb} / \mathrm{MeV} \mathrm{sr})$ & 0.0044 & 0.0607 & 0.0388 \\
\hline & $B$ & 5.39 & 3.33 & 4.55 \\
\hline \multirow[t]{2}{*}{200} & $A(\mathrm{mb} / \mathrm{MeV} \mathrm{sr})$ & 0.0005 & 0.0069 & 0.0188 \\
\hline & $B$ & 7.6016 & 5.63 & 4.84 \\
\hline
\end{tabular}

TABLE VI. Parametrizations of the high energy neutrons in the reactions $p(800 \mathrm{MeV})+\mathrm{Al}(\mathrm{Zr}, \mathrm{Pb})$.

\begin{tabular}{|c|c|c|c|c|}
\hline $\begin{array}{c}E_{n}^{p} \\
(\mathrm{MeV}) \\
\end{array}$ & $00 \mathrm{MeV})+$ & $\mathrm{Al}$ & $\mathrm{Zr}$ & $\mathrm{Pb}$ \\
\hline \multirow[t]{2}{*}{105} & $A(\mathrm{mb} / \mathrm{MeV} \mathrm{sr})$ & 0.0457 & 0.2434 & 0.5261 \\
\hline & $B$ & 1.25 & 0.71 & 0.49 \\
\hline \multirow[t]{2}{*}{125} & $A(\mathrm{mb} / \mathrm{MeV} \mathrm{sr})$ & 0.0434 & 0.0839 & 0.4948 \\
\hline & $B$ & 1.41 & 2.30 & 1.44 \\
\hline \multirow[t]{2}{*}{150} & $A(\mathrm{mb} / \mathrm{MeV} \mathrm{sr})$ & 0.0212 & 0.0625 & 0.1813 \\
\hline & $B$ & 2.46 & 2.98 & 1.92 \\
\hline \multirow[t]{2}{*}{200} & $A(\mathrm{mb} / \mathrm{MeV} \mathrm{sr})$ & & 0.0614 & 0.1813 \\
\hline & $B$ & & 1.95 & 1.92 \\
\hline \multirow[t]{2}{*}{300} & $A(\mathrm{mb} / \mathrm{MeV} \mathrm{sr})$ & 0.0053 & 0.0182 & 0.0496 \\
\hline & $B$ & 3.46 & 3.42 & 2.30 \\
\hline \multirow[t]{2}{*}{400} & $A(\mathrm{mb} / \mathrm{MeV} \mathrm{sr})$ & 0.0014 & 0.0079 & 0.0417 \\
\hline & $B$ & 5.05 & 4.04 & 2.42 \\
\hline \multirow[t]{2}{*}{500} & $A(\mathrm{mb} / \mathrm{MeV} \mathrm{sr})$ & 0.0012 & 0.0075 & 0.0186 \\
\hline & $B$ & 4.89 & 3.78 & 3.76 \\
\hline \multirow[t]{2}{*}{600} & $A(\mathrm{mb} / \mathrm{MeV} \mathrm{sr})$ & $2.9 \times 10^{-5}$ & $3.3 \times 10^{-5}$ & 0.0063 \\
\hline & $B$ & 9.43 & 10.00 & 4.38 \\
\hline \multirow[t]{2}{*}{700} & $A(\mathrm{mb} / \mathrm{MeV} \mathrm{sr})$ & & & 0.0015 \\
\hline & $B$ & & & 6.66 \\
\hline
\end{tabular}




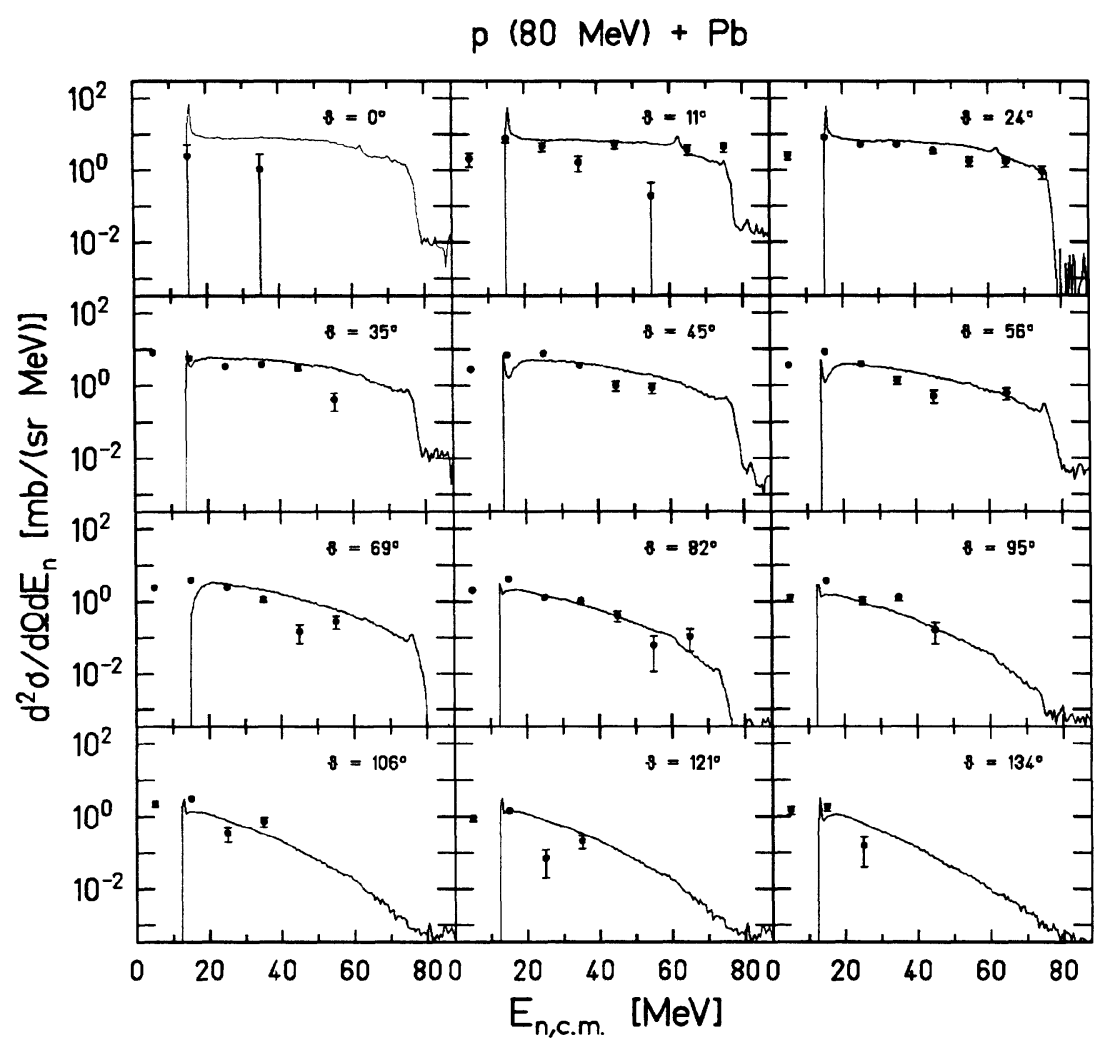

FIG. 15. Same as Fig. 11 for the reaction $p(80 \mathrm{MeV})+\mathrm{Pb}$.

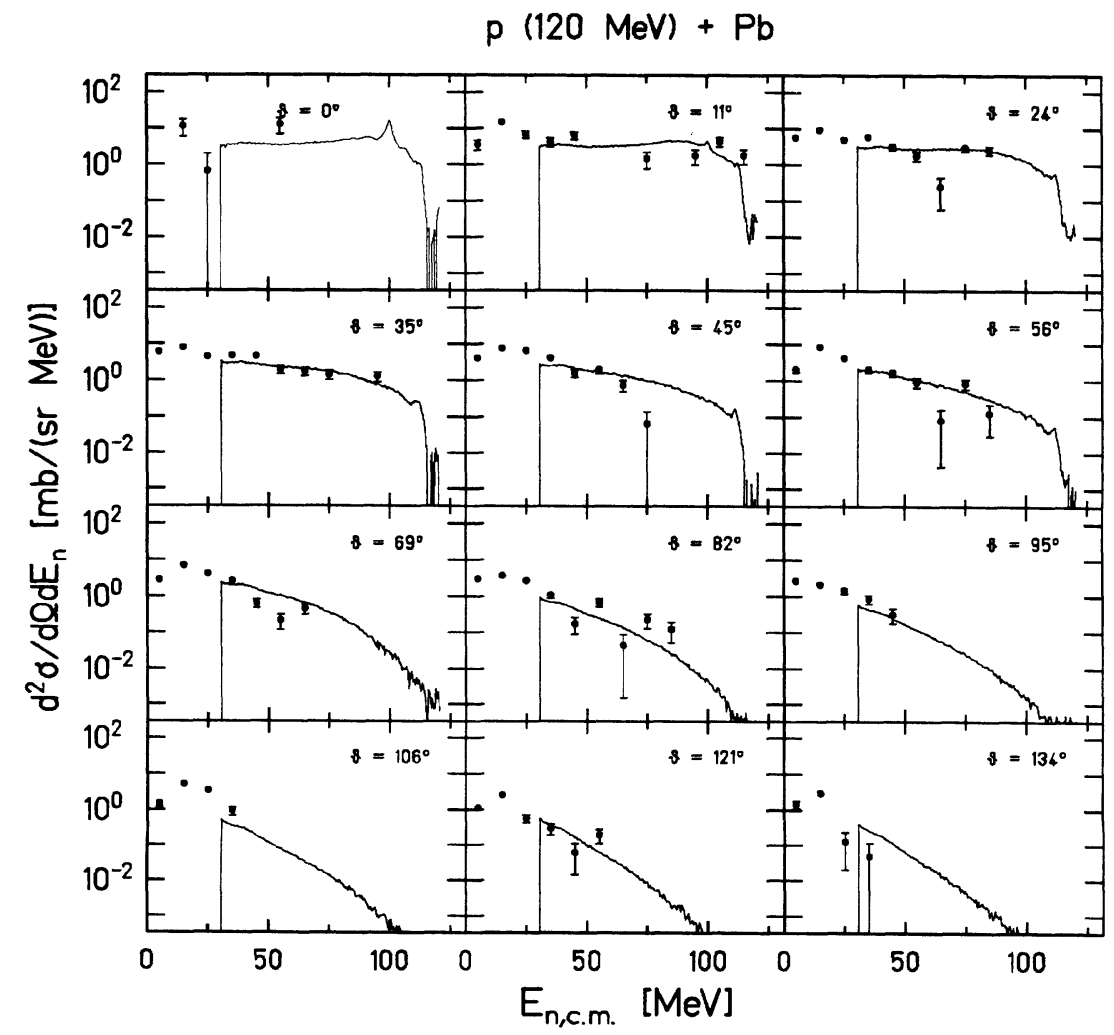

FIG. 16. Same as Fig. 11 for the reaction $p(120 \mathrm{MeV})+\mathrm{Pb}$. 


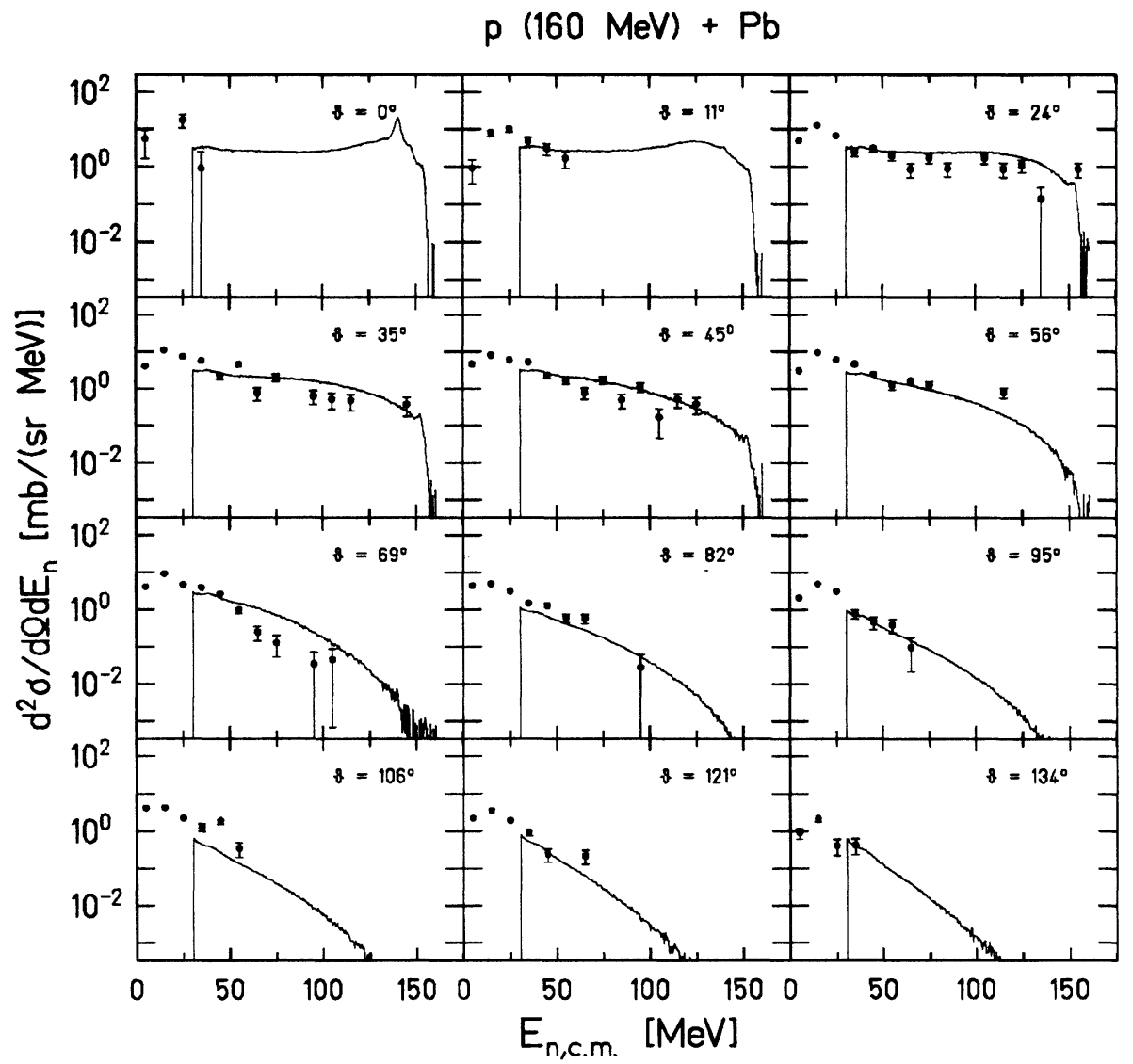

FIG. 17. Same as Fig. 11 for the reaction $p(160 \mathrm{MeV})+\mathrm{Pb}$.

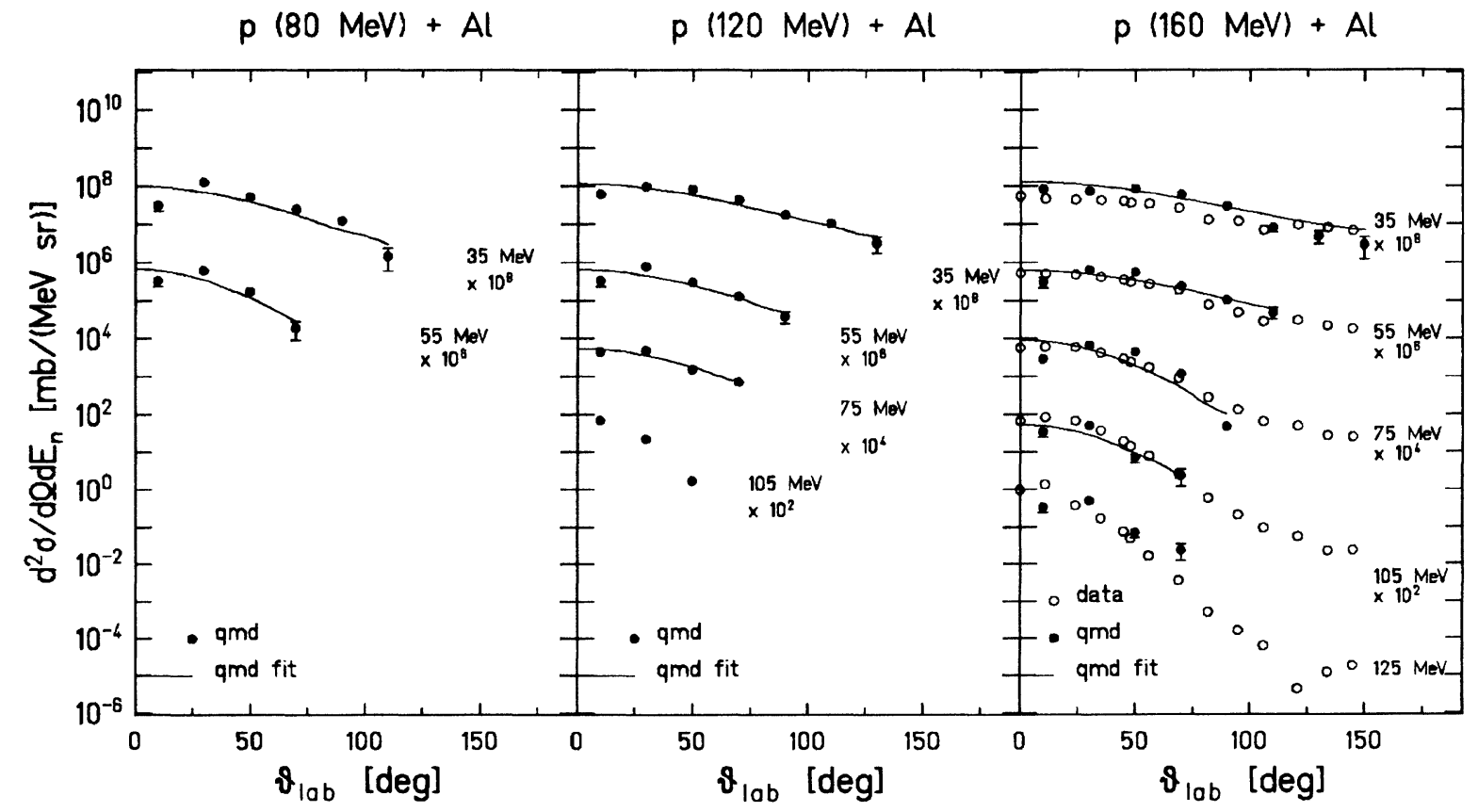

FIG. 18. Angular distributions for the $p+\mathrm{Al}$ reactions for neutrons with different c.m. energies as indicated. The QMD results (full symbols) are compared to the data (open symbols). The lines indicate a parametrization of the QMD results of the form $d^{2} \sigma / d \Omega d E_{n}=A \exp (B \cos \theta)$. 


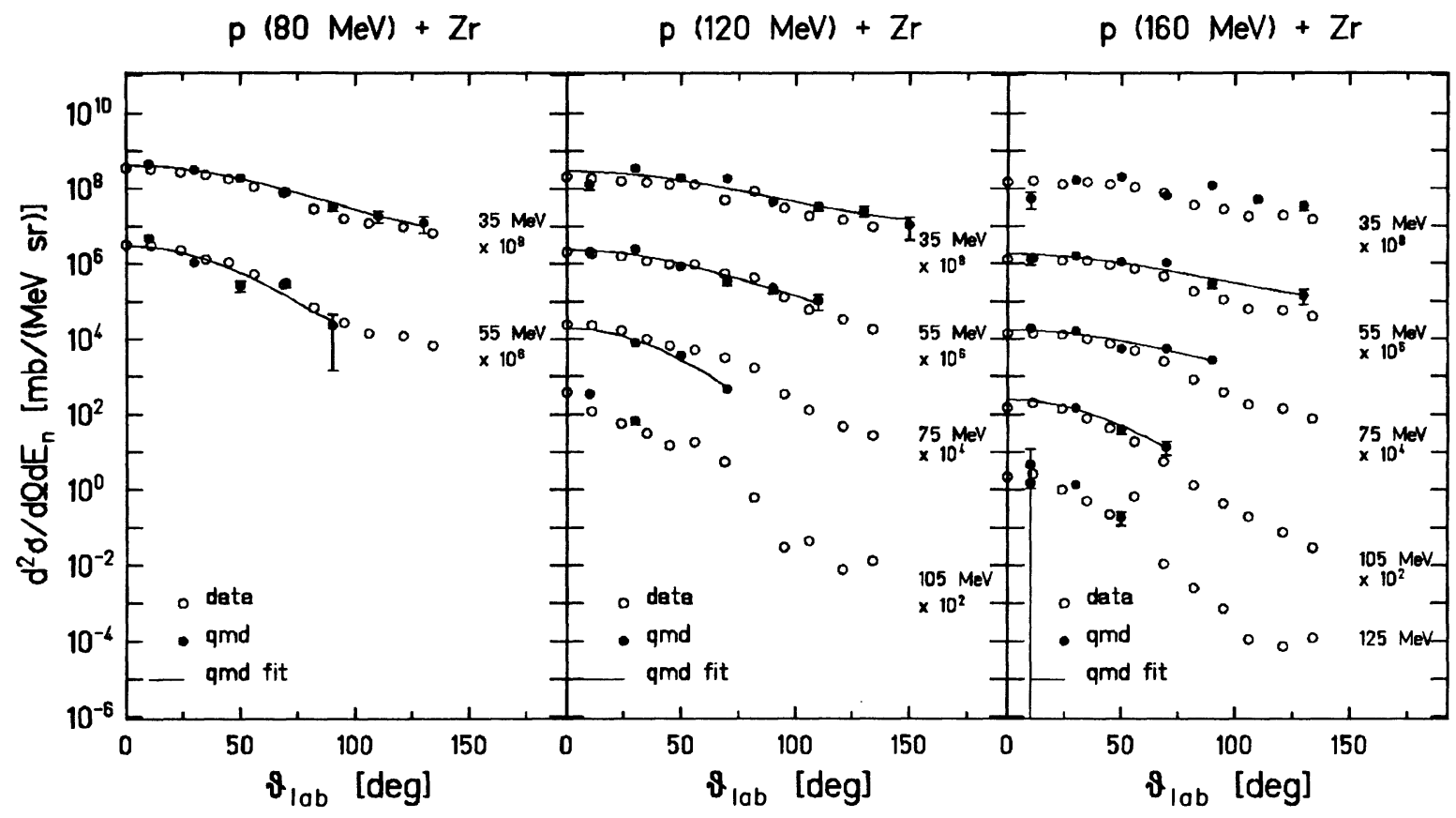

FIG. 19. Same as Fig. 18 for the $p+\mathrm{Zr}$ reactions.

Metropolis sampling procedure. Because of the complicated nature of the Pauli potential, we introduced two different parametrizations of the Pauli potential, where either the total or the kinetic energy alone compares with the exact energy of a free Fermi gas. Using the many- body Hamiltonian of the QMD model we reproduce the proper binding energies and root-mean-square radii of finite nuclei.

The inclusion of the Pauli potential into the QMD model yields well-defined Fermionic ground states.

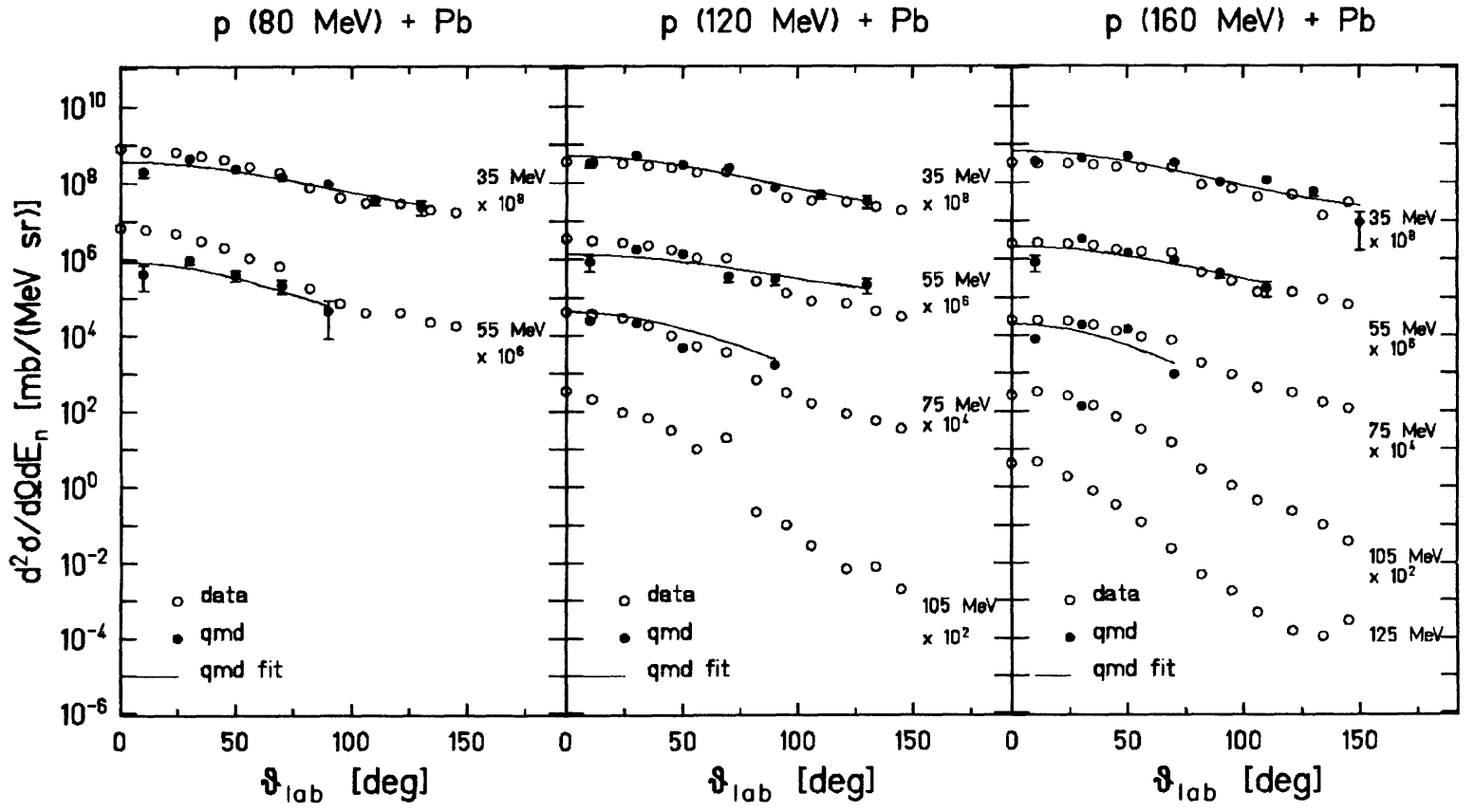

FIG. 20. Same as Fig. 18 for the $p+\mathrm{Pb}$ reactions. 
Therefore it is also possible to give the excitation energy of the emitted fragments.

We have shown how the deexcitation of hot and compressed nuclei takes place in this model. The model is shown to reproduce the fast nucleon cascade dynamics quite well by comparisons of calculated and experimental double-differential spectra for a broad range of incident energies and target masses.

\section{ACKNOWLEDGMENTS}

This work was supported in part by the German Federal Minister for Research and Technology (BMFT), the Deutsche Forschungsgemeinschaft (DFG), and the Gesellschaft für Schwerionenforschung (GSI). We thank Prof. W. Scobel for supplying us with the experimental data.
[1] W. Scheid, H. Müller, and W. Greiner, Phys. Rev. Lett. 32, 741 (1974).

[2] H. G. Baumgardt, J. U. Schott, Y. Sakamoto, E. Schopper, H. Stöcker, J. Hofmann, W. Scheid, and W. Greiner, Z. Phys. A 273, 359 (1975).

[3] H. Stöcker, J. A. Maruhn, and W. Greiner, Phys. Rev. Lett. 44, 725 (1980); Z. Phys. A 290, 297 (1979).

[4] H. Stöcker, L. P. Csernai, G. Graebner, G. Buchwald, H. Kruse, R. Y. Cusson, J. A. Maruhn, and W. Greiner, Phys. Rev. Lett. 47, 1807 (1981); Phys. Rev. C 25, 1873 (1982).

[5] G. Buchwald, G. Gräbner, J. Theis, J. A. Maruhn, W. Greiner, and H. Stöcker, Phys. Rev. Lett. 52, 1594 (1984).

[6] H. Stöcker and W. Greiner, Phys. Rep. 137, 277 (1986).

[7] H.-A. Gustafsson, H. H. Gutbrod, B. Kolb, H. Löhner, B. Ludewigt, A. M. Poskanzer, T. Renner, H. Riedesel, H. G. Ritter, A. Warwick, F. Weik, and H. Wieman, Phys. Rev. Lett. 52, 1590 (1984).

[8] R. E. Renfordt, D. Schall, R. Bock, R. Brockmann, J. W. Harris, A. Sandoval, R. Stock, H. Ströbele, D. Bangert, W. Rauch, G. Odyniec, H. G. Pugh, and L. S. Schröder, Phys. Rev. Lett. 53, 763 (1984).

[9] D. Beavis, S. Y. Chu, S. Y. Fung, W. Gorn, A. Huie, D. Keane, J. J. Lu, R. T. Poe, B. C. Shen, and G. VanDalen, Phys. Rev. C 27, 2443 (1983).

[10] H. G. Ritter, K. G. R. Doss, H. A. Gustafsson, H. H. Gutbrod, K. H. Kampert, B. Kolb, H. Löhner, B. Ludewigt, A. M. Poskanzer, A. Warwick, and H. Wieman, Nucl. Phys. A447, 3c (1985).

[11] K. G. R. Doss, H. A. Gustafsson, H. H. Gutbrod, K. H. Kampert, B. Kolb, H. Löhner, B. Ludewigt, A. M. Poskanzer, H. G. Ritter, H. R. Schmidt, and H. Wieman, Phys. Rev. Lett. 57, 302 (1986).

[12] K. G. R. Doss, H. A. Gustafsson, H. H. Gutbrod, J. W. Harris, B. V. Jacak, K. H. Kampert, B. Kolb, A. M. Poskanzer, H. G. Ritter, H. R. Schmidt, L. Teitelbaum, M. Tincknell, S. Weiss, and H. Wieman, Phys. Rev. Lett. 59, 2720 (1987).

[13] B. V. Jacak, G. D. Westfall, G. M. Crawley, D. Fox, C. K. Gelbke, L. H. Harwood, B. E. Hasselquist, W. G. Lynch, D. K. Scott, H. Stöcker, M. B. Tsang, G. Buchwald, and T. J. M. Symons, Phys. Rev. C 35, 1751 (1987).

[14] K. H. Kampert, J. Phys. G 15, 691 (1989).

[15] H. H. Gutbrod, A. M. Poskanzer, and H. G. Ritter, Rep. Prog. Phys. 52, 1267 (1989).

[16] H. H. Gutbrod, K. H. Kampert, B. W. Kolb, A. M. Poskanzer, H. G. Ritter, and H. R. Schmidt, Z. Phys. A 337, 57 (1990).

[17] H. R. Schmidt, GSI Report 91-03, 1991.

[18] B. Friedman and V. R. Pandharipande, Nucl. Phys. A361, 502 (1981)

[19] G. Bertsch and P. J. Siemens, Phys. Lett. 126B, 9 (1983).

[20] P. J. Siemens, Nature 305, 410 (1983).
[21] D. Hahn and H. Stöcker, Nucl. Phys. A476, 718 (1988).

[22] A. L. Goodman, J. I. Kapusta, and A. Z. Mekjian, Phys. Rev. C 30, 851 (1984).

[23] A. D. Panagioutou, M. W. Curtin, H. Toki, D. K. Scott, and P. J. Siemens, Phys. Rev. Lett. 52, 496 (1984).

[24] L. P. Csernai and J. I. Kapusta, Phys. Rep. 131, 223 (1986).

[25] M. E. Fisher, Physics 3, 255 (1967).

[26] J. E. Finn, S. Agarval, A. Bujak, J. Chuang, L. J. Gutay, A. S. Hirsch, A. T. Minich, N. T. Porile, R. P. Scharenberg, B. C. Stringfellow, and F. Turkot, Phys. Rev. Lett. 49, 1321 (1982); R. W. Minich, S. Agarwal, A. Bujak, J. Chuang, J. E. Finn, L. J. Gutay, A. S. Hirsch, N. T. Porile, R. P. Scharenberg, and B. C. Stringfellow, Phys. Lett. 118B, 458 (1982).

[27] B. V. Jacak, G. D. Westfall, C. K. Gelbke, L. H. Harwood, W. G. Lynch, D. K. Scott, H. Stöcker, M. B. Tsang, and T. L. M. Symons, Phys. Rev. Lett. 51, 1846 (1983).

[28] A. I. Warwick, H. H. Wieman, H. H. Gutbrod, M. R. Maier, J. Peter, H. G. Ritter, H. Stelzer, F. Weik, M. Friedman, D. J. Henderson, S. V. Kaufman, E. P. Steinberg, and B. D. Wilkins, Phys. Rev. C 27, 1083 (1983).

[29] K. Aleklett, W. Loveland, M. De Saint-Simon, L. Sihver, J. O. Liljenzin, and G. T. Seaborg, Nucl. Phys A499, 591 (1982); Phys. Lett. B 236, 404 (1990).

[30] C. K. Gelbke and D. Boal, Prog. Part. Nucl. Phys. 19c, 33 (1987).

[31] J. Pochodzalla, W. Trautmann, and U. Lynen, Phys. Lett. B 232, 41 (1989).

[32] R. Trockel, U. Lynen, J. Pochodzalla, W. Trautmann, N. Brummund, E. Eckert, R. Glasov, K. D. Hildenbrand, K. H. Kampert, W. F. D. Müller, D. Pelte, H. J. Rabe, H. Sann, R. Santo, H. Stelzer, and R. Wafa, Phys. Rev. Lett. 59, 2844 (1987); R. Trockel, K. D. Hildenbrand, U. Lynen, W. F. D. Müller, H. J. Rabe, H. Sann, H. Stelzer, W. Trautmann, R. Wada, E. Eckert, J. Pochodzalla, and N. Brummund, Phys. Rev. C 38, 576 (1988); 39, 279 (1989).

[33] B. Jakobsson, G. Jonsson, B. Lindkvist, and A. Oskarsson, Z. Phys. A 307, 293 (1982).

[34] C. J. Waddington and P. S. Freier, Phys. Rev. C 31, 888 (1985).

[35] J. P. Alard, J. Arnold, J. Augerat, R. Babinet, N. Bastid, F. Brochard, J. P. Costilhes, M. Crouau, N. De Marco, M. Drouet, P. Dubieux, H. Fanet, Z. Fodor, L. Fraysse, J. Girard, P. Gorodetzky, J. Gosset, C. Laspalles, M. C. Lemaire, D. L.'Hote, B. Lucas, G. Montarou, A. Pauneau, M. J. Parizet, J. Poitou, C. Racca, W. Schimmerling, J. C. Tamain, Y. Terrien, J. Valero, and O. Vallette, Nucl. Instrum. Methods A261, 379 (1987).

[36] C. A. Ogilvie, D. A. Cebra, J. Clayton, S. Howden, J. Karn, A. Vander Molen, G. D. Westfall, W. K. Wilson, and J. S. Winsfield, Phys. Rev. C 40, 654 (1989).

[37] C. A. Ogilvie, J. C. Adloff, M. Begemann-Blaich, P. 
Bouissou, J. Hubele, G. Imme, I. Iori, P. Kreutz, G. J. Kinde, S. Leray, V. Lindenstruth, Z. Liu, U. Lynen, R. J. Meijer, U. Milkau, W. F. J. Müller, C. Ngo, J. Pochodzalla, G. Raciti, G. Rudolf, H. Sann, A. Schüttauf, W. Seidel, L. Stuttge, W. Trautmann, and A. Tucholski, Phys. Rev. Lett. 67, 1214 (1991).

[38] D. R. Bowman, G. F. Peaslee, R. T. de Souza, N. Curlin, C. K. Gelbke, W. G. Gong, Y. D. Kim, M. A. Lisa, W. G. Lynch, L. Phair, M. B. Tsang, C. Williams, N. Colonna, K. Hanold, M. A. McMahan, G. J. Wozniak, L. G. Moretto, and W. A. Friedman, Phys. Rev. Lett. 67, 1527 (1991).

[39] J. P. Alard et al., Phys. Rev. Lett. 69, 889 (1992).

[40] J. Randrup and S. E. Koonin, Nucl. Phys. A356, 223 (1981); S. E. Koonin and J. Randrup, ibid. A474, 173 (1987).

[41] G. Fai and J. Randrup, Nucl. Phys. A381, 557 (1982); A404, 551 (1983).

[42] D. H. E. Gross and X. Z. Zhang, Phys. Lett. 161B, 47 (1985); D. H. E. Gross, X. Z. Zhang, and S. Y. Xu, Phys. Rev. Lett. 56, 1544 (1986); D. H. E. Gross, Rep. Prog. Phys. 53, 605 (1990).

[43] J. P. Bondorf, R. Donangelo, I. N. Mishustin, C. J. Pethick, H. Schulz, and K. Sneppen, Nucl. Phys. A443, 321 (1985); J. P. Bondorf, R. Donangelo, I. N. Mishustin, and H. Schulz, ibid. A444, 460 (1985).

[44] A. S. Botvina, A. S. Iljinov, I. N. Mishustin, J. P. Bondorf, R. Donangelo, and K. Sneppen, Nucl. Phys. A475, 663 (1987); A. S. Botvina, A. S. Iljinov, and I. N. Mishustin, ibid. A507, 649 (1990).

[45] D. Hahn and H. Stöcker, Nucl. Phys. A476, 718 (1988).

[46] M. Blann, Phys. Rev. Lett. 54, 2215 (1985); Phys. Rev. C 32, 1231 (1985).

[47] M. Blann, T. Komoto, and I. Tservaya, Phys. Rev. C 40, 2498 (1989).

[48] M. Blann, M. G. Mustafa, G. Peilert, H. Stöcker, and W. Greiner, Phys. Rev. C 44, 431 (1991).

[49] C. Barbagallo, J. Richert, and P. Wagner, Z. Phys. A 324, 97 (1986).

[50] H. Kruse, B. V. Jacak, and H. Stöcker, Phys. Rev. Lett. 54, 289 (1985).

[51] J. J. Molitoris, H. Stöcker, and B. L. Winer, Phys. Rev. C 36, 220 (1987).

[52] H. Kruse, B. V. Jacak, J. J. Molitoris, G. D. Westfall, and H. Stöcker, Phys. Rev. C 31, 770 (1985).

[53] G. F. Bertsch, H. Kruse, and S. Das Gupta, Phys. Rev. C 29, 673 (1984).

[54] J. Aichelin and H. Stöcker, Phys. Lett. 163B, 59 (1985).

[55] J. Aichelin and G. F. Bertsch, Phys. Rev. C 31, 1730 (1985).

[56] C. Gale, G. F. Bertsch, and S. Das Gupta, Phys. Rev. C 35, 1666 (1987).

[57] G. F. Bertsch, W. G. Lynch, and M. B. Tsang, Phys. Lett. B 189, 384 (1987).

[58] C. Gregoire, B. Remaud, F. Sebille, L. Vinet, and Y. Raffray, Nucl. Phys. A465, 317 (1987).

[59] V. Koch, B. Blättel, W. Cassing, and U. Mosel, Nucl. Phys. A532, 715 (1991).

[60] A. Bodmer and C. N. Panos, Phys. Rev. C 15, 1342 (1977).

[61] J. J. Molitoris, J. B. Hoffer, H. Kruse, and H. Stöcker, Phys. Rev. Lett. 53, 899 (1984).

[62] L. Wilets, E. M. Henley, M. Kraft, and A. D. MacKellar, Nucl. Phys. A282, 341 (1977).

[63] L. Wilets, Y. Yariv, and R. Chestnut, Nucl. Phys. A301,
359 (1978).

[64] D. J. E. Callaway, L. Wilets, and Y. Yariv, Nucl. Phys. A327, 250 (1979).

[65] A. R. Bodmer, C. Panos, and A. D. MacKellar, Phys. Rev. C 22, 1025 (1980).

[66] S. M. Kiselev and Yu. E. Pokroskil, Yad. Fiz. 38, 82 (1983) [Sov. J. Nucl. Phys. 38, 46 (1983)].

[67] S. M. Kiselev, Yad. Fiz. 39, 32 (1984) [Sov. J. Nucl. Phys. 39, 18 (1984)].

[68] S. M. Kiselev, Yad. Fiz. 44, 946 (1986) [Sov. J. Nucl. Phys. 44, 610 (1986)].

[69] T. J. Schlagel and V. R. Pandharipande, Phys. Rev. C 36, 162 (1987).

[70] J. Aichelin and H. Stöcker, Phys. Lett. B 176, 14 (1986).

[71] J. Aichelin, G. Peilert, A. Bohnet, A. Rosenhauer, H. Stöcker, and W. Greiner, Phys. Rev. C 37, 2451 (1988).

[72] G. Peilert, A. Rosenhauer, J. Aichelin, H. Stöcker, and W. Greiner, Mod. Phys. Lett. A 3, 459 (1988); Phys. Rev. C 39, 1402 (1989).

[73] A. Bohnet, J. Aichelin, G. Peilert, H. Stöcker, and W. Greiner, Phys. Rev. C 44, 2111 (1991).

[74] M. Berenguer, C. Hartnack, G. Peilert, J. Aichelin, H. Stöcker, and W. Greiner, J. Phys. G 18, 655 (1992).

[75] J. Konopka, Diploma thesis, University Frankfurt (1991).

[76] G. Peilert, Ph.D. thesis, University Frankfurt (1992), GSI Report 92-13, 1992.

[77] J. Aichelin, Phys. Rep. 202, 233 (1991).

[78] G. E. Beauvis, D. H. Boal, and J. C. K. Wong, Phys. Rev. C 35, 645 (1987).

[79] D. H. Boal and J. N. Glosli, Phys. Rev. C 38, 1870 (1988); 38, 2621 (1988).

[80] D. H. Boal, J. N. Glosli, and C. Wicentowich, Phys. Rev. C 40, 601 (1989).

[81] C. Dorso and J. Randrup, Phys. Lett. B 215, 611 (1988); 232, 29 (1989).

[82] Y. Yariv and Z. Fraenkel, Phys. Rev. C 20, 2227 (1979); 24, 488 (1981).

[83] K. K. Gudima and V. D. Toneev, Yad. Fiz. 27, 67 (1978) [Sov. J. Nucl. Phys. 27, 351 (1978)]; K. K. Gudima, H. Iwe, and V. D. Toneev, J. Phys. G 5, 229 (1979).

[84] J. Cugnon, T. Mizutani, and J. Vandermeulen, Nucl. Phys. A352, 505 (1981); J. Cugnon, Phys. Rev. C 22, 1885 (1980).

[85] G. Peilert, J. Randrup, H. Stöcker, and W. Greiner, Phys. Lett. B 260, 271 (1991).

[86] N. Metropolis, A. W. Metropolis, M. N. Rosenbluth, A. H. Teller, and E. Teller, J. Chem. Phys. 21, 1087 (1953).

[87] M. G. Mustafa, M. Blann, A. V. Ignatyuk, and S. M. Grimes, Phys. Rev. C 45, 1078 (1992).

[88] H. Feshbach, A. Kerman, and S. E. Koonin, Ann. Phys. 125, 429 (1980).

[89] M. Blann and H. K. Vonach, Phys. Rev. C 28, 1475 (1983).

[90] M. Blann, W. Scobel, and E. Plechaty, Phys. Rev. C 30, 1493 (1984).

[91] H. Gruppelaar, P. Nagel, and P. E. Hodgson, Nuovo Cimento 9, 1 (1986).

[92] M. Trabandt, W. Scobel, M. Blann, B. A. Pohl, R. C. Byrd, C. C. Foster, S. M. Grimes, and R. Bonetti, Phys. Rev. C 39, 452 (1989).

[93] C. Kalbach, Phys. Rev. C 37, 2350 (1988).

[94] W. Scobel, M. Trabandt, M. Blann, B. A. Pohl, B. A. Remington, R. C. Byrd, C. C. Foster, R. Bonetti, C. Chiese, and S. M. Grimes, Phys. Rev. C 41, 2010 (1990). 